\title{
Le savoir est une fête ! \\ Entretien avec Christian Jacob
}

Réalisé par Jean-François Bert ${ }^{1}$, Jérôme Lamy ${ }^{2}$ et Arnaud Saint-Martin ${ }^{3}$

Il y a mille manières de faire de la recherche en sciences humaines et sociales. De l'enquête obsidionale sans cesse remise sur l'établi à la grande synthèse englobante, les choix déterminent un certain style de savoir. Christian Jacob procède d'une manière peu courante: il emprunte les voies de traverse qui mènent de l'Antiquité grecque à l'anthropologie des savoirs; il trace lui-même les chemins hésitants qui relient l'érudition philologique et la diffusion numérique des connaissances; il ouvre des pans entiers de recherche (e.g. la cartographie comme fait social), indique des pistes, fournit des problématiques avant d'explorer un autre domaine.

Cet entretien est l'occasion de revenir sur la carrière de Christian Jacob, les rencontres déterminantes, les choix thématiques et les enjeux épistémologiques qui ont été les siens. L'historien de la périégèse, frotté de grec et de latin, s'est d'abord essayé à la sémiotique greimassienne, puis il a rencontré Jean-Pierre Vernant et son groupe de recherche réuni au sein du Centre Louis Gernet. L'anthropologie historique des manières de faire, des façons de connaître s'ouvre alors comme un champ de recherche particulièrement fécond. La cartographie grecque offre un terrain d'exploration remarquablement dense. L'apprentissage auprès de ces maîtres généreux que sont Vernant, Vidal-Naquet et Detienne, est d'abord la célébration renouvelée du savoir construit en commun, de la discussion simultanément bienveillante et exigeante. Et Christian Jacob de poursuivre, alors qu'il est entré au CNRS, sa tâche d'éclaireur éclectique. Outre la géographie antique, ce sont désormais tous les savoirs qui sont l'objet de son attention. L'entretien est l'occasion de revenir en détail sur la monumentale tétralogie des Lieux de savoir, entreprise mutilée puisque deux volumes seulement sont parus en 2007 et 2011. Les labyrinthiques arcanes de l'édition française obligent l'historien à rompre les lances pour récupérer son projet. Aujourd'hui relancé, via la plateforme Savoirs, le dispositif prend des allures de Babel digitale, cherchant la circulation entre les grandes aires de savoir, sans rien sacrifier à la précision des contextes, des époques et des lieux. Contre une certaine hyperspécialisation ${ }^{4}$ des disciplines, le nouvel objet autour duquel s'active Christian Jacob n'est que la continuation numérique de sa grande traversée des savoirs.

\section{Greimas et Pausanias}

Zilsel - Tu es entré à l'École Normale Supérieure en 1976 et tu obtiens l'agrégation de lettres classiques en 1980. As-tu commencé à étudier directement l'histoire antique ? Pourquoi avoir choisi de travailler sur Pausanias en maîtrise ? Quel était l'horizon des études sur l'Antiquité à l'époque ? Connaissais-tu déjà le travail d'anthropologie historique mené au sein Centre Louis Gernet, par Jean-Pierre Vernant notamment?

Christian Jacob - Quand je rentre à l'École Normale Supérieure (ENS, rue d'Ulm) en 1976, j'ai en tête de préparer le concours de l'École Française d'Athènes (EFA) et de me spécialiser en archéologie grecque - c'est une passion que j'avais depuis le lycée. En terminale, un de mes enseignants, ancien élève de l'EFA, m'avait dit: "Si tu veux faire de l'archéologie, il faut d'abord passer ton bac, faire une classe préparatoire et entrer à l'ENS ". Il avait raison, et je lui

\footnotetext{
1 Université de Lausanne (Suisse), Institut d'histoire et anthropologie des religions, JeanFrancois.Bert@unil.ch.

${ }^{2}$ CNRS, CERTOP, jerome.lamy@laposte.net.

${ }^{3}$ CNRS, CESSP, arnaud.saint-martin@cnrs.fr.

${ }^{4}$ Steven Shapin, "Hyper-Professionalism and the Crisis of Readership in the History of Science ", Isis, vol. $96, n^{\circ} 2,2005$, p. 238-243.
} 
suis reconnaissant de m'avoir donné une feuille de route. Lors de ma deuxième année de khâgne au lycée Louis-le-Grand, j'ai la chance de suivre les cours d'André Pessel, qui a marqué des générations d'élèves par son enseignement de la philosophie. C'est une discipline que je découvre réellement à ce moment-là. Pessel délivre un enseignement qui n'est pas du tout académique : il nous invite à penser par nous-mêmes, à dépasser le caractère académique des exercices pour faire œuvre de réflexion personnelle. II me suggère de lire Jean-Pierre Vernant, notamment Mythe et pensée chez les Grecs ${ }^{5}$ et Les origines de la pensée grecque ${ }^{6}$. C'est un coup de foudre intellectuel immédiat. Cela me change du thème et de la version grecs, de l'explication littéraire des textes, même si les cours d'histoire ancienne déploient déjà un horizon social, culturel, politique et religieux beaucoup plus large! Je réussis le concours d'entrée à l'ENS à la deuxième tentative, en 1976, grâce notamment à une très bonne note en philosophie, que je dois à la formation émancipatrice de Pessel, et indirectement à Jean-Pierre Vernant et à Marcel Detienne. Le sujet de dissertation était le suivant : "Les rapports entre le théorique et le pratique ". J'ai fait une dissertation en extrapolant à partir des Ruses de I'intelligence, la mètis des Grecs ${ }^{7}$, en évoquant la marche du crabe, le coup de main des chasseurs et pêcheurs, la boiterie d'Héphaïstos, les sophistes et Socrate. Rétrospectivement, je pense que ce devoir de concours a eu un rôle matriciel dans tout mon parcours intellectuel sur I'anthropologie historique des savoirs, puisqu'au fond, je continue de creuser cette question des rapports entre pratique et théorie. Ce sujet bateau a donc été le point de départ d'une très longue croisière...

Je trouve rue d'Ulm un climat de grande liberté intellectuelle, le sentiment d'être au seuil d'un parcours aventureux, où toutes les directions possibles s'offrent à moi. Je suis à la fois des cours techniques d'archéologie grecque (épigraphie, sculpture, céramique, architecture) et les séminaires de Marcel Detienne et Jean-Pierre Vernant. J'ai cette idée un peu folle d'essayer de croiser les fils de l'archéologie et de l'anthropologie historique du monde grec. Je commence un mémoire de maîtrise avec deux directeurs. Le premier est Philippe Bruneau, professeur d'archéologie grecque à Paris IV. Ses séminaires sont non conventionnels, iconoclastes. Très influencé par la linguistique de Jean Gagnepain, Bruneau accompagne son enseignement de l'archéologie par une forte exigence de réflexivité critique et de théorie. II sera l'un des pionniers de l'archéologie du monde moderne, au croisement des sciences sociales. C'était un grand "Délien ", c'est-à-dire un spécialiste du site de Délos, où il avait fouillé. Le codirecteur, c'est Marcel Detienne, qui sera l'un de mes maîtres et principaux interlocuteurs intellectuels pendant les années qui suivront. Nous convenons que je vais passer mon année à travailler sur la Description de la Grèce (ou Périégèse) de Pausanias, un texte que j'avais découvert, quelques années auparavant, lorsque mon père m'avait ramené de Londres les volumes à couverture verte de l'édition Loeb. J'ai fait cette maîtrise en deux années, ce que me permettait le cursus à l'ENS. Parallèlement à mon intérêt pour la Grèce ancienne, j'étais aussi très séduit par ce qui attirait nombre de jeunes chercheurs à l'époque, la sémiotique de Julien Algirdas Greimas, que je percevais alors comme un structuralisme de combat et comme une machine théorique pouvant aplanir tous les terrains et tous les objets. J'ai donc écrit une première version de mon mémoire dans une perspective très sémiotique, très jargonnante et sans doute insupportable de formalisme cuistre : Pausanias et le discours de l'espace. Ce texte a fait hurler Marcel Detienne; Philippe Bruneau, quant à lui, m'a dit une phrase dont je me souviens encore: "Soit vous êtes le dernier des escrocs intellectuels, soit vous êtes le gars brillant de votre génération ". C'est quelque chose qui me hante encore, et je n'ai toujours pas la réponse à cette alternative. Ils $\mathrm{m}^{\prime}$ ont donc proposé soit de soutenir immédiatement - parce que j'avais quand même réalisé un travail sérieux -, mais en m'orientant vers la sémiotique et en oubliant l'EFA, soit de faire une contre-épreuve, en prenant la dernière édition du Guide bleu de la Grèce, en appliquant les algorithmes, les équations et les carrés logiques de

\footnotetext{
${ }^{5}$ Jean-Pierre Vernant, Mythe et pensée chez les Grecs. Études de psychologie historique, Paris, Maspero, 1965.

${ }^{6}$ Jean-Pierre Vernant, Les origines de la pensée grecque, Paris, Presses universitaires de France, 1962.

${ }^{7}$ Marcel Detienne et Jean-Pierre Vernant, Les ruses de l'intelligence. La mètis des Grecs, Paris, Flammarion, 1974.
} 
Greimas, et de voir si j'obtenais ou non les mêmes résultats que dans mon travail sur Pausanias. C'est que j'ai fait et je me suis rendu compte que j'arrivais effectivement aux mêmes résultats. Nous avons donc convenu que je prenais une nouvelle année pour reprendre tout mon mémoire dans une perspective différente. Cette fois l'influence de Detienne fut beaucoup plus forte, il m'a appris à travailler, à poser des questions et à les contextualiser dans la bibliothèque et la culture antiques. Je pris alors mes distances avec la sémiotique, mais aussi avec l'archéologie. Pour être franc, je trouvais que le milieu de mes condisciples, enfin, certains d'entre eux, candidats à l'EFA était horripilant : me retrouver dans cette ambiance de compétition, de concurrence, de carriérisme et de clientélisme à l'égard des mandarins qui détenaient les clés du concours m'était insupportable.

J'avais entre-temps découvert ce lieu improbable qu'était le Centre de Recherches Comparées sur les Sociétés Anciennes, futur Centre Louis Gernet, et aujourd'hui refondu dans I'UMR Anhima, avec des maîtres qui proposaient une approche neuve de l'enseignement et de la recherche et qui me permettaient de renouer avec mon intérêt pour la philosophie. L'exigence et le plaisir intellectuels ont alors pris le pas sur tout le reste, y compris sur le souci de faire une carrière " académique ».

J'opère donc une bifurcation vers Marcel Detienne, Jean-Pierre Vernant, Pierre Vidal-Naquet et le collectif de jeunes chercheurs qui les entourait. Nous sommes à la fin des années 1970. Jean Bousquet, archéologue et spécialiste d'épigraphie grecque, directeur de l'ENS à l'époque, l'a très mal pris. Malgré tout, j'ai été soutenu par les caïmans de l'ENS, qui ont été tout à fait remarquables et $m^{\prime}$ ont toujours encouragé. Je pense toujours avec reconnaissance à des enseignants comme Jean Lallot, Alain Le Boulluec et Dominique Briquel : ils m'ont fait un peu souffrir dans les cours de thème grec ou latin pour la préparation de l'agrégation, mais ils ont été des guides intellectuels d'une grande bienveillance dans mes premiers cheminements de recherche et mon engagement dans l'équipe de Vernant.

Je n'ai pas publié mon mémoire de maîtrise sur Pausanias, seulement quelques articles, dont I'un publié dans la prestigieuse revue Yale French Studies, mais qui fut traduit d'une manière totalement délirante ${ }^{8}$ : par exemple, j'évoque la "logique des qualités sensibles " chez LéviStrauss, et cela devient "la logique des qualités susceptibles". C'était l'un de mes premiers articles, la traduction anglaise ne m'avait pas été communiquée, je n’ai jamais mentionné ce texte dans ma bibliographie!

Ce premier travail de recherches sur la Description de la Grèce de Pausanias m'a permis de mettre au point une approche, une méthode, un type de lecture que j'ai appliqué par la suite à d'autres textes antiques : aborder le texte étudié dans sa globalité, du début à la fin, en faire une lecture lente, en prenant des notes, en balisant, en laissant émerger les idées et hypothèses, en étant sensible à la "musique " des mots et du discours : récurrences, leitmotives, lexique, champs sémantiques, marqueurs énonciatifs, citations... Pour Pausanias, ce sont dix livres à explorer, au ras du texte. Ce corpus constitue depuis longtemps la bible de chevet des archéologues. C'est une description qui produit un tel effet de réel qu'elle semble ne pouvoir appeler qu'une utilisation documentaire, une lecture positiviste, où le texte serait transparent à ses contenus. J'y ai vu une forme de défi ! J'ai choisi de considérer le texte complet comme un discours, comme un système, et je l'ai déconnecté de la critique de son exactitude par rapport au savoir archéologique contemporain. Je lui ai appliqué une méthode de lecture influencée par la sémiotique, mais surtout orientée vers des questionnements plus anthropologiques. Je me demandais en le lisant: "Quel est le projet de cet auteur, ce contemporain de Marc Aurèle, qui décide de mettre la Grèce continentale en rouleaux, qui se déplace de cité en cité, d'une région à l'autre, qui note tout ce qu'il voit, les monuments, les statues, qui transcrit les histoires qu'on lui raconte, qui parsème un peu de son érudition lettrée et antiquaire par-dessus, et qui trace par le fil de son écriture un parcours géométrique et cartographique dans lequel les villes deviennent les points nodaux de réseaux régionaux complexes? ». Pausanias effectue un travail extraordinaire de tissage de la topographie, par une grammaire des repérages, des orientations, des contiguïtés déterminées par son parcours,

\footnotetext{
${ }^{8}$ Christian Jacob, « The Greek traveler's areas of knowledge: Myths and other discourses in Pausanias Description of Greece », Yale French Studies, n 59,1980 , p. 65-85.
} 
son regard, même si ceux-ci sont in fine des constructions rétrospectives et systématiques, une fois les voyages réalisés, une fois venu le temps de l'écriture : il crée un espace discursif qui peut aussi bien concerner une cité, une région, un monument ou des images...

Ce travail de maîtrise m'a donc sensibilisé à la géographie, à la sémiotique de l'espace, à la cartographie. D'autres thèmes sont apparus, que j'ai continué à travailler par la suite: I'histoire des projets intellectuels, la formation des discours, le statut de l'énonciation dans les textes savants et la construction des savoirs. Déjà à l'époque j'avais fait de nombreuses fiches sur la problématique du couplage entre symphonie et diaphonie des savoirs. J'avais remarqué que Pausanias était capable d'intégrer dans son récit des controverses qui lui étaient rapportées en jouant sur les discordances et les accords de ses informateurs. La force d'une symphonie (ou d'une diaphonie) de témoignages autour d'un énoncé m'avait impressionné.

La soutenance de ma maîtrise s'est finalement bien passée, avec Detienne et Bruneau. Je poursuis dans le même temps mes explorations archéologiques en m'intéressant au domaine protohistorique dans le monde égéen et la Crète : j'avais commencé à apprendre l'écriture du linéaire $B$ et cette langue syllabique qui est un proto-grec. La protohistoire me semblait un domaine plus rigoureux que l'archéologie du monde classique où prédominaient encore un humanisme un peu nébuleux et les schémas esthétisants de l'histoire de l'art. Toutefois, l'attrait intellectuel du groupe de Vernant et Detienne fut le plus fort. L'archéologie protohistorique, c'était lire le sol, la terre, les pierres, les tessons de céramique, les ossements des sépultures. L'anthropologie historique des mondes anciens, c'était lire une bibliothèque, une culture, des univers intellectuels et imaginaires. Dans leur sillage, ces maîtres charismatiques ont attiré une première génération de jeunes chercheurs, comme Jeannie Carlier, Alain Schnapp, Annie Schnapp, Nicole Loraux, Françoise Frontisi, Florence Dupont, Pauline Schmitt, Jesper Svenbro, Pierre Ellinger, Jean-Louis Durand, François Lissarague, François Hartog. Chacun, dans cette génération, prenait de gros risques pour sa carrière en venant travailler avec Vernant, Vidal Naquet, Detienne. Ils savaient que les recrutements à l'université étaient difficiles, qu'il y avait une forte opposition, politique, intellectuelle, des milieux des études anciennes. On m'avait mis en garde : "Tu sais, tu es normalien, tu as des chances de faire une belle carrière universitaire, ce serait peut-être bien que tu reviennes dans cinq ou dix ans, en étant professeur ". Mais j'ai refusé, j'étais alors très "pur et dur » et je voulais absolument travailler dans ce cadre intellectuellement enthousiasmant, sans faire de compromis de carrière... Je ne le regrette pas...

Zilsel - Qu'est-ce qui te pousse vers ces objets d'anthropologie historique? Comment s'est forgée cette curiosité pour l'Antiquité grecque?

Christian Jacob - J'avais un grand-père professeur de lettres classiques, que je n'ai pas connu, mais j'ai hérité d'une partie de sa bibliothèque. Dès la quatrième, les cours de grec ont été, pour moi, une révélation. Je ne m'intéressais pas à la physique, à la chimie et aux mathématiques : je ne voulais faire que du grec, ce qui $m^{\prime}$ a valu quelques frictions avec mes parents et mes professeurs. J'avais déjà un fond de textes grecs et latins assez conséquent, des éditions du début du $20^{\mathrm{e}}$ siècle, couvertes des annotations à l'encre de mon grand-père. Une de mes premières lectures fut l'Alceste d'Euripide, j'avais réussi à lire une tragédie en entier, en cherchant les mots dans le dictionnaire Bailly, en me faisant un petit carnet de vocabulaire et un autre de grammaire, que j'apprenais par cœur! Lorsque je me suis lancé dans mes premières recherches, j'ai voulu m'écarter des approches purement littéraires : je ne voulais pas faire le énième mémoire sur l'éternel humain chez Euripide ou Sophocle. Je voulais travailler sur des auteurs moins connus, explorer des terrae incognitae... Pausanias et sa Périégèse de la Grèce m'ont fasciné. À la lecture de cette description, on "voit » les rues, les monuments, les temples, tels qu'ils étaient au $2^{\mathrm{e}}$ siècle ; on suit quelqu'un qui prend des notes, qui documente son voyage, qui fait des enquêtes de terrain, qui archive des données, c'est absolument passionnant. Cette fascination m'a conduit à déconstruire cet " effet de réel » et d'immédiateté pour comprendre comment il était produit, pour entrer dans la fabrique même de ce discours et de ce savoir antiquaires... Pausanias se fait l'ethnographe, voire l'anthropologue de sa propre culture, à la fois immergé dans celle-ci, mais aussi avec une distance critique et réflexive. Et au fond, cette position d'intériorité/extériorité constitue un fil conducteur de toutes mes recherches, sur Alexandrie, sur Denys le Périégète, sur Athénée de 
Naucratis, sur Lucien, mais aussi sur les savoirs, puisque je suis à la fois un observateur et un sujet observé, à travers les miroirs de la réflexivité.

J'ai été influencé par les travaux de Michel de Certeau sur l'espace, notamment dans $L^{\prime \prime I n v e n t i o n ~ d u ~ q u o t i d i e n ~}{ }^{9}$, mais aussi ses écrits sur Jean de Léry ${ }^{10}$ : son approche des récits de voyage et de la "rhétorique piétonnière " a été une source d'inspiration majeure, et son analyse des deux modèles de discours de l'espace, basés sur la carte ou l'itinéraire, offrait un cadre méthodologique opératoire. Lire Pausanias, pour le jeune normalien que j'étais, c'était plonger dans une érudition foisonnante, celle de l'auteur que j'étudiais, mais aussi celle qu'il fallait mobiliser pour le comprendre et le contextualiser ; c'était aussi mettre en œuvre un style de recherche, une méthodologie qui, avant l'avènement des outils informatiques, imposaient des voyages au long cours (j'ai passé plusieurs mois à mettre le texte en fiches, des fiches de bristol rangées par thèmes dans des boîtes en bois !). J'étais engagé dans un corps à corps avec un texte que peu de chercheurs avaient vraiment lu pour lui-même, du début à la fin, de sa surface vers ses couches profondes. Je faisais un travail très mécanique de prospection, d'inventaire, de classement. II me fallait entendre la musique du texte et laisser émerger les thèmes, les assonances, les rythmes des mots et des concepts. Je pense que les requêtes instantanées permises par l'informatique sur des textes numérisés ne remplacent pas cette lecture lente, ce corps-à-corps avec une œuvre ou un corpus...

J'avais vraiment le sentiment d'être fait pour ce type de recherche; je savais que j'en avais la vocation, que j'aimais voir surgir des hypothèses, des idées, m'immerger dans ce travail patient et exigeant qui permettait de renouveler, de personnaliser l'interprétation d'un texte. J'étais très idéaliste à l'époque - je pense que je le suis encore. J'ai renoncé à l'EFA. Je savais que travaillant dans l'équipe de Vernant, je n'aurais aucune chance sur des postes de grec en université, dont l'accès était soigneusement filtré. J'ai passé l'agrégation de lettres classiques deux années de suite, à l'époque il y avait très peu de postes... J'ai encore des cauchemars aujourd'hui à propos de ce concours... J'ai finalement obtenu mon agrégation et disons que les difficultés ont vraiment commencé après. Au début des années 1980, ma sortie de l'École se profilait. II fallait nommer un caïman d'histoire ancienne rue d'Ulm, les enseignants de l'École m'ont proposé le poste, mais les gens de Paris IV y ont mis leur veto : ils ne voulaient pas d'un élève de Detienne, Vernant et Vidal-Naquet dans cette position. Le directeur de l'ENS littéraire à ce moment-là, le géographe Marcel Roncayolo, qui était un directeur d'études à l'EHESS, m'était favorable, mais il a dû céder. J'ai pris conscience alors que les mondes académiques n'étaient pas des communautés iréniques. Rétrospectivement, je n'ai pas de regret. Ce poste impliquait un lourd travail de préparation à l'agrégation d'histoire, même s'il m'aurait permis de donner une grande visibilité à l'anthropologie historique des mondes antiques à l'École. Je crois d'ailleurs avoir été l'un des rares normaliens de cette génération à m'orienter vers l'équipe de Vernant, avec Alain Ballabriga, et un peu plus tard Arnaud Zucker, ou encore François de Polignac, qui venait de l'ENS de Saint-Cloud.

\section{Un fort assiégé ?}

Zilsel - Il y avait donc des clivages puissants au sein des études grecques. Quels en étaient les ressorts politiques et scientifiques?

Christian Jacob - II y avait des enjeux politiques très puissants. On connaît le combat courageux de Vidal-Naquet contre la torture durant la guerre d'Algérie, pour établir la vérité sur la disparition de Maurice Audin ${ }^{11}$, engagement qui lui vaudra de perdre son poste universitaire pendant un an en 1960. Vernant fut membre du Parti Communiste Français (PCF) jusqu'en 1969 et fut aussi un signataire du Manifeste des 121 en faveur de l'insoumission lors de la guerre d'Algérie. Ils étaient l'un et l'autre très marqués à gauche, même si Vernant avait l'aura d'un Compagnon de la Libération, et l'establishment universitaire leur était hostile (certains professeurs de grec et de latin ne cachaient pas des sympathies très droitières).

\footnotetext{
${ }^{9}$ Michel de Certeau, L'invention du quotidien, T. 1 : Arts de faire, Paris, Union Générale d’Édition, 1980.

${ }^{10}$ Michel de Certeau, L'Écriture de l'histoire, Paris, Gallimard, 1975, p. 215-248.

${ }^{11}$ Pierre Vidal-Naquet, L'Affaire Audin (1957-1978), Paris, Minuit, 1989.
} 
Et puis surtout, les recherches qu'ils menaient, leurs publications chez des éditeurs engagés et "grand public ", comme François Maspero, remettaient en cause la pratique des études grecques telles qu'on les concevait à Paris IV et aussi la circulation des travaux savants, qu'ils rendaient accessibles et pertinents pour un public beaucoup plus large et diversifié que celui des spécialistes. En mobilisant l'approche anthropologique, en assumant le fait que le sens n'est jamais donné et qu'il faut toujours interpréter et construire, ils créaient également une vraie rupture avec les méthodes d'histoire et de commentaire littéraires empreintes de subjectivité et de poncifs humanistes ou se limitant à une approche philologique et stylistique des textes. D'autant que notre petit groupe était très perméable aux influences de la linguistique et de l'anthropologie structurales, telles que les pratiquait par exemple Claude Lévi-Strauss, pour apporter un éclairage radicalement nouveau sur les textes grecs, dont l'altérité et la complexité étaient revendiquées pour nourrir un défi herméneutique, faire surgir de nouveaux objets, de nouvelles interprétations. Que l'on pense par exemple au texte de Vernant sur le mythe des races dans les Travaux et les Jours d'Hésiode ${ }^{12}$, au livre de Detienne sur Les Jardins d'Adonis. La mythologie des aromates en Grèce ancienne ${ }^{13}$.

Ma propre expérience scolaire $m^{\prime}$ avait déjà sensibilisé à ce hiatus. Lorsque j'ai validé ma licence de lettres classiques à la Sorbonne, j'ai dû passer trois fois le certificat de littérature française. En effet, en passant par la khâgne et l'hypokhâgne, j'avais été encouragé à lire les ouvrages de Gérard Genette, Jean-Pierre Richard, Charles Mauron, Lucien Goldman, Roland Barthes, autant de grands noms de la "nouvelle critique ", extraordinairement stimulants. Mais à Paris IV, de telles références provoquaient un rejet épidermique. Comme du reste les noms de Vernant ou Detienne dans un oral de grec. Même si I'on ne citait pas explicitement ces auteurs, si on avait le malheur d'employer les mots de "structure ", de "diégèse ", de "dispositif énonciatif ", voire d' "énonciation", (sans même parler des "isotopies" ou des " carrés logiques » et des " actants » de Greimas), immédiatement la sanction tombait... Je me suis ainsi fait coller deux fois de suite au certificat de littérature française à Paris IV. À la troisième tentative (j'étais en deuxième année rue d'Ulm), après mon explication de texte, j'ai carrément dit aux examinateurs de l'épreuve orale: "Écoutez, je vous promets de ne plus jamais remettre les pieds à la Sorbonne, je fais du grec, je ne ferai pas de littérature française, vous ne me reverrez plus, mais je vous en prie, je suis en deuxième année d'ENS, donnez-moi ce certificat pour que je puisse soutenir ma maîtrise. " J'ai eu juste la moyenne, mais j'ai obtenu mon certificat. Tout cela parce que la formation littéraire des khâgnes incitait à interroger les textes avec des catégories très innovantes, et à ne pas se contenter de commentaires esthétisants et subjectifs. Certains cours d'agrégation à Paris IV étaient consternants. Comme j'ai préparé ce concours deux ans de suite, j'ai découvert à ma grande stupeur qu'un professeur de latin réputé faisait à peu près le même cours d'une année sur l'autre, sur deux auteurs différents: il employait les mêmes ficelles rhétoriques, c'était le même plan, les mêmes poncifs qui revenaient. En grec, Jean Bousquet, directeur de l'ENS, nous faisait étudier I'lon d'Euripide en se contentant de lire quatre lignes de grec, quatre lignes de traduction du volume Budé, avec des commentaires affligeants. Je suis d'ailleurs parti en plein cours tellement tout cela était lamentable, et méprisant à l'égard d'agrégatifs, dont le temps était précieux... Je me souviens que Nicole Loraux avait eu la générosité de me donner le manuscrit d'une étude encore inédite sur cette tragédie, offrant des clés de lecture autrement pertinentes. L'intelligence, l'exigence intellectuelle, la générosité étaient une nouvelle fois du côté de l'équipe de Vernant...

Je devais donc faire face à des choix intellectuels et politiques, entre une voie royale, où il fallait faire la cour à des mandarins, dont tous n'étaient pas respectables, pour progresser dans le cursus et entrer dans le moule de la reproduction académique (la lecture de Bourdieu,

\footnotetext{
12 Jean-Pierre Vernant, "Le mythe hésiodique des races. Essai d'analyse structurale ", Revue d'histoire des religions, $\mathrm{T} .157, \mathrm{n}^{\circ} 1,1960$, p. 21-54.

${ }^{13}$ Marcel Detienne, Les jardins d'Adonis. La mythologie des parfums et des aromates en Grèce, Paris, Gallimard, 1972.
} 
Homo academicus ${ }^{14}$, plus tard, m'a semblé décrire tout cela d'une manière lumineuse), et une voie plus exigeante, un peu plus risquée aussi, mais, au fond, sans compromis.

Zilsel - Quelle était l'ambiance à l'ENS au moment où tu prépares l'agrégation ? Y avait-il une concurrence entre sciences sociales et philosophie ? Et comment as-tu tracé ta trajectoire dans cet espace des possibles?

Christian Jacob - L'ENS a été pour moi une formidable matrice intellectuelle, un lieu de découvertes et de liberté. Dès la rentrée rue d'Ulm, en septembre 1976, je souhaite suivre le séminaire de philosophie de Louis Althusser. À la première séance, il fait un tour de salle et je lui explique que je veux être helléniste, mais que j'ai un intérêt personnel pour la philosophie que je veux continuer à développer. II m'a répondu, en me foudroyant du regard: "Ici on ne veut pas d'amateurs ". Je ne suis jamais retourné à son séminaire. Je n'ai toujours pas compris la réaction d'Althusser, mais il est trop tard pour en discuter avec lui...

Les sciences sociales sont arrivées à l'ENS à la fin de ma scolarité. Je pense que j'ai raté quelque chose, parce que j'étais déjà plongé dans ma thèse à ce moment-là. Marcel Roncayolo, le directeur de l'École littéraire, me dit : "Vous allez organiser un séminaire chaque semaine, on essaie de mettre en place un DEA de sciences sociales". Je l'ai fait, mais j'ai compris plus tard, trop tard, que c'était sans doute un moyen de me mettre sur les rails pour I'EHESS. Mais les choses n'ont pas vraiment pris à ce moment-là.

Entre-temps Vidal-Naquet $m^{\prime}$ avait obtenu un poste de pensionnaire-normalien à la Bibliothèque Nationale, au département des Cartes et Plans. J'avais aussi fait une candidature à la Fondation Thiers, j'ai été reçu par Jacqueline de Romilly qui, en regardant mon dossier sur lequel il était écrit que je travaillais avec Detienne et Vernant, me demanda : «Est-ce que vous avez quelque chose à dire en votre faveur ? " Je n'étais ni pupille de la Nation, ni mutilé de guerre, ni lauréat du Concours général... " Non, Madame, je n'ai rien à dire... " Je n'ai donc pas obtenu la Fondation Thiers. L'année suivante, je me suis présenté au CNRS, même si je savais que je n'avais aucune chance, mais c'était pour faire un tour de piste. Ce fut probablement l'un des moments les plus surréalistes de ma carrière. Devant la commission, je fais un petit exposé sur les géographes grecs. En face de moi, François Chamoux, professeur d'histoire grecque, vraisemblablement mon rapporteur, $m^{\prime}$ accable de reproches et $m^{\prime}$ humilie publiquement: "Quand on a un dossier aussi faible et une aussi mauvaise connaissance des textes grecs, on ne se présente pas au CNRS ! ". Etc. Cela a duré un bon quart d'heure. C'était méchant et blessant. L'idée de renverser la table et de quitter la salle d'audition $\mathrm{m}^{\prime}$ a traversé l'esprit. Je ne I'ai pas fait. Les autres membres du jury, parmi lesquels se trouvaient Jacques Jouanna et François Jouan, n'ont pas bronché. Chamoux était congestionné de colère. Je suis sorti de cette audition livide, anéanti. Quelques jours plus tard, je me rends au bureau de la Revue des Études Grecques, à Paris IV - je faisais des comptes rendus pour avoir des livres gratuits. Je rentre dans la pièce, François Chamoux est là. Je suis pétrifié de trouille. Mais il ne me reconnaît pas. II me demande sur quoi je travaille. Je lui explique que je commence un travail sur les géographes grecs. II me répond : "C'est très bien, mais ne faites pas comme ce Christian Jacob qui étudie avec Marcel Detienne et Jean-Pierre Vernant ». Je sors avec une pile de livres, Chamoux me donne des Budés. J'appelle depuis une cabine Vernant et lui raconte la scène... II rit et me dit que Chamoux, pendant l'audition au CNRS, a dû être tellement aveuglé par la haine, à la simple vue des lettres de recommandation qui accompagnaient mon dossier, qu'il ne m'a probablement même pas regardé. Tout cela avait une dimension politique forte, car Chamoux était très marqué à droite. II avait écrit un volume sur la Grèce hellénistique dans lequel il n'évoque jamais les contacts des Grecs avec les Juifs, les Perses et les Égyptiens, ce qui, pourtant, est constitutif de la civilisation hellénistique. Pour lui, la Grèce devait rester dans sa pureté idéale, sans être contaminée par ce que Arnaldo Momigliano a magnifiquement décrit comme les "sagesses barbares ${ }^{15}$. Cet épisode m'a profondément marqué, et je n'ai jamais humilié ni accablé un étudiant ou une étudiante, même lorsque son travail prêtait à la critique. On peut avoir un dialogue intellectuel exigeant, mais sans blesser la personne en face de soi...

\footnotetext{
${ }^{14}$ Pierre Bourdieu, Homo Academicus, Paris, Minuit, 1984.

${ }^{15}$ Arnaldo Momigliano, Sagesses barbares, les limites de l'hellénisation, Paris, Maspero, 1979.
} 
J'ai donc ressenti, dès cette époque, que les études grecques étaient un objet à la fois scientifique et politique, et que le monde académique, loin d'être irénique, était un champ de bataille où les étudiants pouvaient être les victimes collatérales.

\section{L'effervescence du Centre Louis Gernet}

Zilsel - Tu soutiens ta thèse d'histoire grecque en 1987 sous la direction de Pierre-Vidal Naquet. Tu en tires un ouvrage, La description de la Terre habitée de Denys d'Alexandrie ${ }^{16}$. Comment t'es-tu intéressé à la géographie antique ? Quels étaient les enjeux d'une étude de la périégèse grecque conçue dans ses dimensions scolaires, poétiques, savantes, mais aussi politiques?

Christian Jacob - J'ai commencé ma thèse Géographie et culture en Grèce ancienne. Essai de lecture de la Périégèse de la Terre habitée de Denys d'Alexandrie avec Marcel Detienne. Mais lorsqu'elle a dépassé les mille pages, sur ses conseils, j'ai converti cette thèse de troisième cycle en thèse d'État et Pierre Vidal-Naquet, à l'EHESS, a pris le relais pour la direction nominale.

Ces deux maîtres étaient très différents. Detienne avait une grande compétence sur le plan de la philologie et de la technicité des études anciennes. II avait l'habitude de dire : "On peut être créatif, radical et imaginatif sur le plan des interprétations, mais il faut être irréprochable sur le plan de l'érudition. " II fallait obliger les adversaires à se positionner par rapport à des idées et des arguments et ne pas laisser de prise à des arguties mineures. II avait chez lui une bibliothèque de travail impressionnante, tous les textes anciens, la Real Encyclopädie PaulyWissowa. Il lisait mes chapitres de près, et m'apportait énormément d'éléments critiques, de compléments, de références bibliographiques et de pistes. II me recevait chez lui (il habitait alors au pied de Montmartre) en début d'après-midi, et on travaillait jusqu'au moment du dîner, généralement constitué d'un risotto et accompagné d'une bonne bouteille de vin. Son exigence intellectuelle était très forte, il m'incitait toujours à aller plus loin. II était aussi généreux : il m'a permis de publier mes premiers articles et $m^{\prime}$ 'a associé à des colloques qu'il organisait. J'ai été l'un de ses élèves proches, parmi d'autres, comme Cléo Carastro et Arnaud Zucker.

Pierre Vidal-Naquet, lui, était animé par le principe de réalité. II m’a ainsi beaucoup interrogé sur les questions d'imaginaire, de représentation. II m'a fortement encouragé, dans ma thèse, à prendre en compte le contexte social, culturel, politique, idéologique de la description géographique de Denys. Ses séminaires d'histoire ancienne, mais aussi d'historiographie et d'éthique historienne, $m^{\prime}$ ont profondément marqué, en particulier ceux sur le négationnisme et sur les écrits de Faurisson. Pour des doctorant·e.s, c'était une invitation à réfléchir sur le statut de la preuve et de la source, sur la falsification, la fragilité de la vérité et de la mémoire. Il faut dire qu'à ces époques, des thèses négationnistes ont pu être soutenues en université, à Lyon et à Nantes, et qu'il y avait un réel enjeu à contrer les Eichmann de papier ${ }^{17}$. Ces leçons de Vidal-Naquet restent très présentes pour moi, et si j'admets l'existence de régimes de vérité différents, je reste très attaché à ne pas verser dans un relativisme généralisé, et à l'idée même de vérité. La falsification de l'histoire existe, elle peut constituer un objet d'étude, si on déconstruit ses rouages, sa rhétorique, ses ellipses, ses trucages.

Detienne et Vidal-Naquet $m$ 'ont fait confiance et $m^{\prime}$ ont laissé travailler exactement sur ce que je voulais, et comme je le voulais. À aucun moment leur tutorat n'a été prescriptif. Il s'agissait d'un accompagnement bienveillant, attentif, exigeant intellectuellement, qui, bien souvent, prenait la forme d'un dialogue. J'ai donc une dette énorme envers eux. J'ajouterai, parmi mes maîtres, les noms de Michel de Certeau et Louis Marin (j'ai donné mes premiers exposés dans le cadre des écoles d'été et des colloques du Centre de sémiotique d'Urbino). Ces grandes figures intellectuelles étaient généreuses de leur temps et de leur savoir avec les étudiants et jeunes chercheurs. Elles étaient aussi accessibles, d'une grande simplicité d'abord, en particulier à Urbino, où les terrasses de café et les auberges de la campagne environnante se

\footnotetext{
${ }^{16}$ Christian Jacob, La description de la Terre inhabitée de Denys d'Alexandrie ou la leçon de géographie, Paris, Albin Michel, 1989.

${ }^{17}$ Pierre Vidal-Naquet, Les Assassins de la mémoire. "Un Eichmann de papier » et autres essais sur le révisionnisme, Paris, La Découverte, 2005.
} 
prêtaient à une convivialité décontractée, ce qui n'était pas incompatible avec des discussions passionnantes, qui étaient comme les after de séminaires plus formels. J'ai pu rencontrer dans ce cadre Umberto Eco et Jean-François Lyotard, je me souviens de la grande simplicité et bienveillance de ce dernier à l'égard du jeune doctorant que j'étais, et $d u$ tee-shirt affichant un Mickey hilare du premier.

Michel de Certeau fut pour moi, comme pour tant d'autres, un véritable éveilleur. Je mesure la chance d'avoir pu le rencontrer, à la fin des années 1970, alors que j'étais rue d'Ulm. Lorsqu'il était à Paris, Certeau recevait chez lui pendant des journées entières, avec des rendez-vous individuels de trois quarts d'heure. Il accordait la même attention à un professeur très connu et à un jeune doctorant. II m'a beaucoup encouragé, inspiré, non seulement sur des dossiers précis, comme la cartographie, les récits de voyage, mais aussi sur le plan d'une éthique de vie plus générale, qu'il s'agisse de l'engagement dans le travail intellectuel ou d'une transmission bienveillante dans le cadre du métier d'enseignant-chercheur. Après mon premier échec à l'agrégation, il m'avait écrit un mot aussi sage que gentil, depuis l'Université de San Diego, m'exhortant à ne pas tout envoyer valser et à me représenter à ce concours. II avait raison, évidemment...

Zilsel - On imagine, autour de Vernant et de Vidal-Naquet, une forte effervescence intellectuelle doublée d'une mise à l'épreuve de l'érudition. Comment s'organisaient les séminaires? D'une façon ou d'une autre, pouvait-on parler d'un collectif de recherche?

Christian Jacob - Le séminaire de Marcel Detienne à la $V^{e}$ section de l'École Pratique des Hautes Études (EPHE) se tenait dans la salle Marcel Mauss avec ses tables étroites si malcommodes, ses strapontins rabattables, le mardi matin, de 11 heures à 13 heures, si je me souviens bien. II se déroulait selon un scénario immuable. II s'ouvrait sur la reprise et la reformulation des propositions avancées lors de la séance précédente. Puis Detienne avançait dans le dossier en cours, alternant lecture et commentaire des sources, hypothèses et contextualisation, avec son style inimitable de conteur volubile, grands gestes, regard vif, diction expressive. C'est ainsi que j'ai vu s'élaborer certains de ses livres, notamment Dionysos mis à mort ${ }^{18}$ et L'écriture d'Orphée ${ }^{19}$, mais aussi toutes les recherches qui ont conduit au livre collectif La cuisine du sacrifice en pays grec ${ }^{20}$. Ces séminaires donnaient lieu à des échanges passionnants avec les chercheurs du premier cercle... Jean-Pierre Vernant était presque toujours présent aussi. Certaines séances étaient animées par des invités, notamment les chercheurs étrangers, et pour un étudiant comme moi, ce fut l'occasion de découvrir un horizon intellectuel plus large.

Jean-Pierre Vernant continue à incarner pour moi un véritable éthos du travail intellectuel, réunissant simplicité, honnêteté, intelligence, et compagnonnage. Dès que je suis arrivé dans son séminaire, j'ai eu l'impression d'être adopté. Tutoiement d'emblée, empathie, je pense que cet abord direct, simple et chaleureux était un trait constitutif de sa personnalité, remontant peut-être aux camaraderies des réseaux combattants de la Résistance.

Son enseignement, comme celui de Detienne, était une fête intellectuelle. Le cours du Collège de France était un peu plus formel qu'à l'EPHE, avec d'abord la leçon professorale, puis une heure de séminaire, consacrée aux chantiers de recherche, et parfois à des exposés d'invités. C'était un dépaysement perpétuel. Je sortais de mes cours d'agrégation, imprégné de verbes irréguliers et de casse-tête syntaxiques (thèmes grec et latin!), un peu épuisé par les cadres scolastiques de cette préparation au concours, et, au Collège ou à l'EPHE, j'entrais dans un monde où l'on évoquait les loups-garous des montagnes d'Arcadie, les autels des sanctuaires ruisselants de sang après les sacrifices, la fuite des Danaïdes, la " belle mort " du héros homérique, le masque terrifiant de la Gorgone, Dionysos qui rend fou et les Bacchantes qui déchiraient le malheureux Penthée à mains nues: ce n'était pas la même Grèce, mais un monde vivant, étrange et étranger, complexe, fascinant, se prêtant à l'exploration

\footnotetext{
${ }^{18}$ Marcel Detienne, Dionysos mis à mort, Paris, Gallimard, 1977.

${ }^{19}$ Marcel Detienne, L'écriture d'Orphée, Paris, Gallimard, 1989.

${ }^{20}$ Marcel Detienne et Jean-Pierre Vernant (dir.), La cuisine du sacrifice en pays grec, Paris, Gallimard, 1979.
} 
anthropologique... C'était un bain d'intelligence, un shoot de vitamines intellectuelles qui me renforçaient dans ma certitude que c'était dans ce milieu-là que je voulais travailler...

Vernant commençait souvent ses interventions par l'expression "écoute voir ". Dans les séminaires, il reprenait ce qu'avait dit l'intervenant et il le reformulait avec une clarté lumineuse, avec intelligence, en en révélant le potentiel avec une immense générosité, sans jamais se mettre dans une posture de surplomb professoral. Mais à l'écouter, on avait l'impression qu'il avait mieux compris que l'intervenant lui-même le cœur du sujet. J'ai eu cette impression moi-même lors de mon dernier rendez-vous avec Vernant, peut-être en 2006, au moment où nous étions plongés dans le chantier du premier volume des Lieux de savoir ${ }^{21}$. Vernant était malade, avait eu des complications d'une opération chirurgicale, il m'a reçu dans son bureau au Collège, je lui ai proposé de reporter notre entretien, il n'a pas voulu, on a donc discuté. Je lui ai parlé du projet des Lieux de savoir, il m'a écouté avec attention, puis... "Écoute voir... " et il a repris les choses d'une manière lumineuse, en me disant que ce projet se situait à la croisée de l'héritage de la psychologie historique de Meyerson et des régimes de vérité et des épistémès de Foucault, il avait tout compris, il reformulait les choses avec sa vision ample, son intelligence, ouvrant et balisant un espace intellectuel où tout faisait sens... J'aurais aimé enregistrer cet entretien qui m'avait galvanisé... Nous avions pensé à Vernant pour un petit texte d'introduction générale au premier volume des Lieux de savoir, mais il était dans un tel état de souffrance physique que cela m'aurait semblé obscène de le lui demander. Il est mort le 9 janvier 2007, il n'aura donc pas vu ce volume que j'aurais tant aimé lui remettre en main propre, en lui disant ma dette intellectuelle et mon immense affection.

Mais revenons à mes années de doctorat, et à ce compagnonnage au Centre de recherches comparées sur les sociétés anciennes, qui occupait alors le rez-de-chaussée de la Maison d'Auguste Comte, au 10 rue Monsieur-le-Prince. Je me souviens que dès cette époque-là, j'étais très soucieux d'épistémologie et de réflexivité. Et j'ai eu le culot de dire, lors d'une réunion du Centre de Recherche : "Ce serait bien qu'on fasse un séminaire pour expliciter nos méthodes de travail ». Vernant $m^{\prime}$ a répondu avec beaucoup de gentillesse et d'indulgence: "Écoute voir, Christian, la méthode c'est le chemin une fois qu'on l'a parcouru ". Il avait raison, évidemment. Nous étions engagés dans des pratiques de recherche empiriques, qui convergeaient sur une même exigence d'intelligibilité et de rigueur, mais qui ne se nouaient pas dans un dogme partagé. Chacun avait son style. Vernant était inimitable. Aucun de ses disciples n'a fait du Vernant; chacun a trouvé sa propre voie, sa voix aussi.

II y aurait beaucoup à dire sur la voix de Vernant, qui m'émeut profondément encore aujourd'hui, lorsque je l'entends sur une vidéo ou un enregistrement. Lors de ses cours sur l'lliade, sur la belle mort du héros, ou sur Dionysos dans les Bacchantes d'Euripide, il devenait conteur, aède, acteur tragique et il captivait son auditoire, qui tremblait pour Achille ou pour Penthée, dans la plus pure tradition de la catharsis aristotélicienne. On retrouve cette voix, cette prosodie, ce souffle, ce rythme et ces intonations dans les textes de Vernant, par exemple dans L'Univers, les Dieux et les Hommes ${ }^{22}$. Vernant avait également la capacité de faire fleurir, dans les textes qu'il commentait, ce qui s'y trouvait en germe, les significations, la beauté, l'étrangeté, la portée; cela demande beaucoup de modestie, d'expérience et de générosité que de faire fleurir les textes et d'apprendre aux jeunes chercheurs, par l'exemple, cet art de jardiner les cultures antiques. Je crois que c'est l'essence même de notre travail d'enseignant-chercheur.

Zilsel - Comment pourrais-tu caractériser la formation de ce petit groupe autour de Jean-Pierre Vernant ? Quelle était sa morphologie, sa logique de fonctionnement?

Christian Jacob - Il y avait, dans le groupe réuni autour de Vernant, Vidal-Naquet et Detienne, une forte cohérence intellectuelle et humaine qui s'est renforcée avec la création du Centre de Recherches Comparées sur les Sociétés Anciennes, qui est devenu une Unité Mixte de Recherche (UMR) du CNRS. Par la suite, le laboratoire est devenu le Centre Louis Gernet, puis, plus récemment ANHIMA (Anthropologie et Histoire des Mondes Antiques), après la fusion avec le Centre Glotz et la jeune équipe Phéacie. Des chercheuses et chercheurs de ma

\footnotetext{
${ }^{21}$ Christian Jacob (dir.), Lieux de savoirs, T. I : Espaces et communautés, Paris, Albin Michel, 2007.

22 Jean-Pierre Vernant, L'Univers, les Dieux et les Hommes, Paris, Seuil, 1999.
} 
génération ont donc vécu le processus d'institutionnalisation de la recherche, la montée en puissance de l'appareil administratif... Je me souviens du conseil de laboratoire où nous avions discuté, avec Vernant, de ce nouveau modèle des UMR du CNRS, en pesant le pour et le contre. Aujourd'hui, avec l'Agence Nationale de la Recherche, le Haut Conseil de l'Évaluation de la Recherche et de l'Enseignement Supérieur (HCERES), les Communautés d'Universités et Établissements (ComUE), les Écoles universitaires de recherche (EUR), les Initiatives, Laboratoire et Équipements d'excellence (IDEX, LABEX, EQUIPEX), les European Research Council (ERC), etc. Nous sommes entrés dans une ère nouvelle. Je ne peux m'empêcher de penser que cet écosystème managérial et concurrentiel à tous les étages n'aurait pas permis à des Vernant, Foucault, Bourdieu et autres de travailler comme ils l'ont fait et de produire des œuvres majeures et fondatrices.

Le "Centre", comme on l'appelait, s'organisait autour de Vernant. C'était la personne fédératrice, il alliait la légitimité scientifique et une forme d'autorité morale incontestable, une aura et un charisme reposant tant sur sa personnalité chaleureuse que sur la trajectoire unique qui l'avait conduit de la Résistance aux études grecques. Marcel Detienne fut d'abord l'assistant de Vernant avant de devenir directeur d'études à la $V^{e}$ section de l'EPHE. Claude Mossé et Pierre Vidal-Naquet appartenaient aussi à ce premier noyau. II y avait une complémentarité de compétences et d'orientations intellectuelles entre philosophie, anthropologie, philologie et histoire.

Outre leurs propres cours, ces maîtres circulaient souvent d'un séminaire à l'autre, ce qui donnait lieu à des discussions passionnantes et à une cohérence de la recherche. De là sont nées des collaborations intenses. Vidal-Naquet et Vernant ont ainsi écrit souvent ensemble, notamment Mythe et tragédie en Grèce ancienne ${ }^{23}$ et les trois volumes de La Grèce ancienne ${ }^{24}$. Avec Marcel Detienne, Vernant a cosigné un livre magnifique, aujourd'hui un classique, Les ruses de l'intelligence. La mètis des Grecs ${ }^{25}$ et codirigé La cuisine du sacrifice en pays grec ${ }^{26}$. Le travail était vraiment collectif, d'autant que le désir de mener des analyses comparatives encourageait à multiplier les collaborations. C'est ainsi que sont parus les volumes sur les Problèmes de la guerre en Grèce ancienne ${ }^{27}$, les Problèmes de la terre en Grèce en ancienne ${ }^{28}$ ainsi que le livre Divination et rationalité ${ }^{29}$. Autour de Vernant s'est ainsi déployé un réseau de spécialistes de différentes aires culturelles: Jean Bottéro pour la Mésopotamie, Léon Vandermeersch pour la Chine, Charles Malamoud pour I'Inde, Michel Cartry pour l'Afrique. Le Centre de recherches comparées sur les sociétés anciennes a très tôt tissé de multiples liens sur le plan international, avec l'Angleterre (Geoffrey E.R. Lloyd de l'Université de Cambridge), I'Italie (Mario Vegetti à l'Université de Pavie) et les États-Unis (Froma I. Zeitlin qui travaillait sur la tragédie et le féminisme à Columbia, Gregory Nagy à Harvard, Charles Segal à Brown). Ce réseau a permis d'une part la traduction dans différentes langues des livres de Vernant, Detienne, Vidal-Naquet, de multiples échanges académiques, mais aussi la formation de doctorant.e.s étranger.e.s qui ont à leur tour contribué à la diffusion de ces perspectives de recherche. Ce succès international contrastait avec l'hostilité de l'establishment des études grecques en France. II faut relire les comptes rendus des ouvrages publiés dans la mouvance de Vernant dans la Revue des Études Grecques. II y avait des snipers - dont par charité je tairai

\footnotetext{
${ }^{23}$ Jean-Pierre Vernant et Pierre Vidal-Naquet, Mythe et tragédie en Grèce ancienne, T. I et T. II, Paris, La Découverte, 1986.

${ }^{24}$ Jean-Pierre Vernant et Pierre Vidal-Naquet, La Grèce ancienne, T. I : Du mythe à la raison, Paris, Seuil, 1990, Jean-Pierre Vernant et Pierre Vidal-Naquet, La Grèce ancienne, T. II : L'espace et le temps, Paris, Seuil, 1991, Jean-Pierre Vernant et Pierre Vidal-Naquet, La Grèce ancienne, T. III : Rites de passage et transgression, Paris, Seuil, 1992.

${ }^{25}$ Marcel Detienne et Jean-Pierre Vernant, Les ruses de la raison. La mètis des Grecs, op. cit.

${ }^{26}$ Marcel Detienne et Jean-Pierre Vernant (dir.), La cuisine du sacrifice en pays grec, op. cit.

${ }^{27}$ Jean-Pierre Vernant (dir.), Problèmes de la guerre en Grèce antique, Paris, La Haye, Mouton, 1968.

${ }^{28}$ Moses I. Finley (dir.), Problèmes de la terre en Grèce ancienne, Paris, La Haye, Mouton, 1973.

${ }^{29}$ Jean-Pierre Vernant, Léon Vandermeersch, Jacques Gernet, Jean Bottéro, Roland Crahay, Luc Brisson, Jeannie Carlier, Denise Grodzynski et Anne Retel-Laurentin, Divination et Rationalité, Paris, Seuil, 1974
} 
les noms - qui étaient mobilisés à chaque fois que Detienne ou Vernant publiait un livre, pour les dézinguer avec une incroyable mauvaise foi.

Du point de vue d'une sociologie de la transmission des savoirs, il est intéressant de noter que Vernant, Detienne et Vidal-Naquet n'ont quasiment jamais publié dans les collections spécialisées, ni d'ailleurs dans la Revue des Études Grecques. Leurs ouvrages étaient édités chez François Maspero, en format de poche ou en livres à reliure souple, et à prix très abordable. II y a eu ensuite Flammarion, Gallimard et Le Seuil. Par ce choix, ces livres ont eu une circulation beaucoup plus large que des travaux d'érudition classique. Ils s'inscrivaient dans le paysage des sciences humaines et sociales plus que dans les études anciennes stricto sensu. Ces livres étaient aussi remarquables par leurs qualités d'écriture, par leur accessibilité...

Le travail au Centre était collectif. Les jeunes chercheurs étaient associés aux chantiers en cours. C'est ainsi que Detienne m'a fait participer au volume collectif Les savoirs de l'écriture en Grèce ancienne ${ }^{30}$, construit au fil d'une série d'ateliers et de séminaires. Le volume Transcrire les mythologies fut le fruit d'un colloque inoubliable qui s'était tenu en septembre 1992 à Taormina, à l'invitation du maestro Giuseppe Sinopoli $i^{31}$. J'ai essayé, à mon niveau, de reproduire cette dimension collective et interactive dans les projets de recherche que j'ai entrepris, car je pense profondément que nous avons besoin de l'intelligence des autres : la pensée prend son essor dans les échanges, le dialogue, la critique et... dans la prise de risque liée à l'interdisciplinarité et au comparatisme comme aux questionnements fondamentaux. Lorsque Marcel Detienne compare l'assemblée des cités grecques à l'assemblée des Cosaques ou aux formes d'organisation des villes italiennes au Moyen-Âge, cela peut faire hurler les hellénistes purs et durs! Mais il a raison! Detienne n'est pas devenu un spécialiste des Cosaques, mais il a trouvé le spécialiste pour discuter avec lui et écrire le chapitre qui lui a donné à penser ${ }^{32}$. On sait qu'il a théorisé ce comparatisme expérimental et heuristique dans un livre qui reste aujourd'hui encore une source majeure d'inspiration pour moi ${ }^{33}$.

Zilsel - On sent, dans ce que tu racontes de ces séminaires, de ces échanges, une forme de camaraderie intellectuelle très puissante. Est-ce un héritage que tu revendiques?

Je me représente le travail intellectuel comme un jeu de cubes qui prennent appui les uns sur les autres. Chacun apporte une pièce, son savoir, sa compétence, son terrain, son style, mais on peut à la fin construire quelque chose excédant les capacités d'un individu. J'ai appris de Vernant et de Detienne l'art de créer des communautés d'ami.e.s, de collègues, d'interlocutrices-eurs, de camarades, en dehors des rapports de pouvoir ou d'institution, pour les différents chantiers collectifs que j'ai animés, les deux volumes Des Alexandries, les Lieux de savoir, et aujourd'hui Savoirs.

Cela devient de plus en plus difficile d'avoir une démarche désintéressée. Les jeunes chercheuses et chercheurs sont pris·es par des exigences de rentabilité, de bibliométrie, des rapports à remettre chaque année, et bien sûr par la concurrence et les obstacles multiples pour obtenir un premier poste ou avancer dans leur carrière. Le monde universitaire est marqué aujourd'hui par une vraie violence institutionnelle, avec la précarité de nombreux emplois, le faible nombre de bourses doctorales ou postdoctorales, la rareté des postes offerts aux concours de recrutement et souvent l'opacité des critères de décision, parmi de multiples dossiers de niveau équivalent. Sans parler de la concurrence entre les établissements et les regroupements d'établissements, des aléas liés aux demandes de financement, dans le cadre des ANR ou des ERC, avec les concours, les contrats doctoraux, les ANR. Je dois reconnaître que l'interdisciplinarité, les projets collectifs et transversaux sont un luxe que je peux me permettre, du fait que je suis chercheur à plein temps, au CNRS et à l'EHESS, deux institutions

\footnotetext{
${ }^{30}$ Marcel Detienne (dir.), Les savoirs de l'écriture en Grèce ancienne, Lyon, Presses universitaires de Lyon, 1988.

${ }^{31}$ Marcel Detienne (dir.), Transcrire les mythologies. Tradition, écriture, historicité, Paris, Albin Michel, 1994.

${ }^{32}$ Marcel Detienne (dir.), Qui veut prendre la parole, Paris, Seuil, 2003.

${ }^{33}$ Marcel Detienne, Comparer l'incomparable, Paris, Seuil, 2000.
} 
qui me donnent la plus grande liberté intellectuelle. Ce n'est évidemment pas le cas de beaucoup de mes collègues universitaires et, a fortiori, de tou.te-s les jeunes chercheuses et chercheurs en situation de grande précarité du fait des contrats courts, et parfois de l'absence de tout contrat. Pour les plus jeunes, après le doctorat, il est clair que la quête de post-docs et les premières candidatures (ATER, maîtres.ses de conférence, chargé.e.s de recherche) imposent un rythme incompatible avec une recherche libre et sereine, avec des perspectives évolutives.

\section{Au contact des savoirs}

Zilsel - En abordant la question des savoirs, tu as croisé les thématiques de l'épistémologie, de la philosophie et de la sociologie des sciences. Comment as-tu construit ton rapport à ces grandes aires épistémiques consacrées aux sciences, aux pratiques de connaissances, aux opérations cognitives?

Christian Jacob - Une trajectoire de chercheur, ce sont non seulement les moments d'une carrière, des lieux, des rencontres, mais aussi différentes phases réflexives, toutes proportions gardées un peu comme un artiste qui peut traverser différentes " périodes ". Mon travail a certes été déterminé par les différents contextes institutionnels, les communautés dans lesquels je me suis intégré ou que je me suis construit, mais aussi par des lectures, des paliers et des déplacements successifs.

Ma thèse est consacrée à la lecture systématique et contextualisée d'un géographe grec mineur, Denys le Périégète (Ile siècle de notre ère), qui me permet d'ouvrir différentes enquêtes, notamment sur l'imaginaire du regard aérien, sur les procédures de description de l'espace géographique, dans leurs liens avec la tradition cartographique grecque, sur les savoirs littéraires, anthropologiques, religieux, politiques qui viennent se greffer sur ce texte, relevant du genre de la poésie didactique et associant plaisir esthétique et visée pédagogique (le texte a rempli la fonction de manuel de géographie, au-delà de l'Antiquité, puisqu'il est utilisé dans les collèges de Jésuites). Je m'engage donc dans l'archéologie d'un champ de savoir, en partant des textes, des concepts, des argumentations, des technologies intellectuelles. II y a un point aveugle dans ce travail : les cartes géographiques elles-mêmes, puisque nous n'en avons pas de cartes antiques - je mets à part les cartes liées à la Géographie de Ptolémée, à partir de Byzance, et deux artefacts surgis récemment dont l'authenticité est très contestée (mappa di Soleto, carte d'Artémidore).

À ma sortie de l'ENS, j'obtiens un poste de "pensionnaire " au Département des cartes et plans de la Bibliothèque nationale. C'est le cadre idéal pour un doctorant qui a besoin d'accéder à un vaste ensemble de sources, mais c'est aussi l'occasion pour moi de m'immerger au quotidien parmi les cartes anciennes. Je n'étais pas satisfait des histoires de la cartographie qui circulaient alors. Elles étaient trop téléologiques, trop positivistes, trop liées aussi à I'histoire de l'art. De manière étonnante, elles évacuaient les aspects épistémologiques liés à l'objet cartographique, son statut d'artefact, la construction de son pouvoir représentatif, sa performativité, son rôle d'écran pour la projection de multiples savoirs, débordant des cadres de la géographie stricto sensu. Mon travail sur les géographes grecs m'avait sensibilisé à ces aspects théoriques, et je me suis dit qu'il était temps de faire un livre qui répondrait à cette question : qu'est-ce qu'une carte ? C'est à ce moment-là que j'ai rencontré David Woodward et Brian Harley, qui lançaient le monumental projet de The History of Cartography, aux Presses de I'Université de Chicago ${ }^{34}$. Le texte programmatique de ces deux géographes américains,

\footnotetext{
34 John Brian Harley et David Woodward (eds.), The History of Cartography, vol. 1 : Cartography in Prehistoric, Ancient, and Medieval Europe and the Mediterranean, Chicago, The University of Chicago Press, 1987 ; John Brian Harley et David Woodward (eds.), The History of Cartography, vol. 2, Livre 1 : Cartography in the Traditional Islamic and South Asian Societies, Chicago, The University of Chicago Press, 1992 ; John Brian Harley et David Woodward (eds.), The History of Cartography, vol. 2 : Livre 2 : Cartography in the Traditional East and Southeast Asian Societies, Chicago, The University of Chicago Press 1994 ; David Woodward et G. Malcom Lewis (eds.), The History of Cartography, vol. 2, Livre 3 : Cartography in the Traditional African, American, Arctic, Australian, and Pacific Societies, Chicago, The University of Chicago Press, 1998 ; David Woodward (ed.), The History of Cartography, vol. 3 : Cartography in the
} 
dans l'introduction générale au premier volume, m'avait enthousiasmé par sa dimension théorique et critique, nourrie des réflexions de Derrida et de Foucault, situant les cartes au cœur des sciences sociales et leur redonnant toute leur complexité, dans un vaste projet comparatiste et interdisciplinaire. Ce fut pour moi une source d'inspiration profonde et un encouragement majeur à m'engager dans ce projet d'une approche théorique de la cartographie à travers l'histoire, qui fut publié par Albin Michel, sous le titre L'empire des cartes, en $1992^{35}$. Ce n'était pas à proprement parler une histoire de la cartographie, mais plutôt une réflexion engagée dans quatre directions: la matérialité de la carte, son langage graphique, son rapport à l'écriture et au langage, et enfin ses effets imaginaires, intellectuels et sociaux. J'ai bâti cette réflexion à partir d'un vaste ensemble de lectures et de l'observation rapprochée, systématique d'une série de cartes de la tradition européenne qui me semblaient particulièrement suggestives pour mes questionnements. Le plan du livre avait été conçu dès le départ, et je l'ai écrit d'une manière pour ainsi dire modulaire, à partir d'une base de données relationnelles, organisée selon l'enchaînement des paragraphes, sous-parties et parties de chaque chapitre. La construction et la rédaction de ce livre furent un vrai bonheur, tant par le nomadisme intellectuel qu'elles me permirent, de l'histoire de l'art à la psychologie, des techniques de gravure à l'heroic fantasy, de la sémiologie à l'anthropologie, que par la liberté d'un style d'écriture à visée théorique, mais qui laissait aussi une place à la subjectivité et à l'imaginaire. Ma plus grande joie fut de voir ce livre traduit en américain et publié par les Presses de l'Université de Chicago, grâce notamment au soutien de David Woodward ${ }^{36}$.

Je dois ici mentionner un trait de ma personnalité qui conditionne sans doute mes cheminements intellectuels. Je suis quelqu'un qui ne tient pas en place, je n'ai jamais voulu m'en tenir à une spécialité, à un terrain disciplinaire, à un objet d'étude unique. J'aurais pu faire toute ma carrière comme spécialiste de la géographie antique, ou de l'histoire de la cartographie, ou de l'histoire des bibliothèques, ou encore de l'érudition gréco-romaine: écrire dans les revues dédiées, être invité dans les colloques disciplinaires, être reconnu, peutêtre, dans l'un de ces domaines. Mais, au fond, il y a une sorte de dynamique personnelle qui me conduit à aller de l'avant, à ne pas me contenter d'une "rente de situation " ou de la sécurité d'un champ de spécialité bien balisé, à me mettre aussi en jeu et en risque. Je conçois que cela puisse donner une impression de superficialité, voire de franche insolence et inconscience, peut-être même d'une certaine arrogance, lorsque l'on s'aventure sur des terrains si différents, dont chacun suffirait à occuper une vie entière de chercheur. Mais je crois que j'obéis aussi à une vraie logique intellectuelle, en déroulant un fil cohérent, depuis mes premiers travaux sur Pausanias jusqu'à mes chantiers actuels sur les savoirs, qui s'ouvrent sur les humanités numériques et certains aspects des sciences cognitives. II me revient en mémoire ce que Michel de Certeau me disait, quand j'étais étudiant : un chercheur est comme un braconnier et il a le droit de poursuivre un lièvre en sautant par-dessus toutes les clôtures disciplinaires. II y a, je le reconnais, une dose d'impertinence dans cette manière de passer d'un champ à l'autre, mais au fond, c'est ce qui rend une trajectoire de chercheur passionnante... Pour être tout à fait honnête, je dois reconnaître que j'ai pu m'offrir le luxe de cette mobilité du fait de mon statut de chercheur au CNRS, puis de mon poste de directeur d'études cumulant à l'EHESS. Si j'avais été enseignant-chercheur dans le département de grec ou d'histoire ancienne d'une université, ma liberté de mouvement aurait été beaucoup plus contrainte.

C'est au cours de la rédaction de L'empire des cartes que j'ai commencé à lire Bruno Latour. Je lui ai d'ailleurs envoyé le livre à sa parution et nous sommes devenus amis. La science en

European Renaissance, Chicago, The University of Chicago Press, 2007 ; Mark Monmonier (ed.), The History of Cartography, vol. 6 : Cartography in the Twentieth Century, Chicago, The University of Chicago Press, 2015.

${ }^{35}$ Christian Jacob, L'empire des cartes. Approche théorique de la cartographie à travers l'histoire, Paris, Albin Michel, 1992.

${ }^{36}$ Christian Jacob, The Sovereign Map: Theoretical Approaches in Cartography Throughout History, Chicago, The University of Chicago Press, 2006. 
action $^{37} \mathrm{~m}$ 'a permis de comprendre bien des aspects de la géographie grecque après coup, en particulier les ressorts des controverses qui traversent cette tradition, d'Eratosthène à Ptolémée, et les stratégies de construction d'un savoir largement conjectural, reposant sur l'autorité et le crédit symbolique de ses acteurs et de leurs sources. La problématique des centres de calcul, des mobiles immuables et la magnifique analyse de la cartographie de la presqu'île de Sakhaline, suite à l'expédition de La Pérouse, m'avaient enthousiasmé. La vie de laboratoire ${ }^{38}$ me fit découvrir le champ de l'ethnographie des pratiques scientifiques, ce fut l'un des points de départ d'une nouvelle phase de mon travail sur les pratiques lettrées, puis savantes, qui m'a conduit aux Lieux de savoir. Je me souviens des séminaires informels et conviviaux que Bruno Latour organisait chez lui, en petit comité, autour d'un livre (on a ainsi discuté Karl Polanyi et d'autres). C'était un laboratoire d'idées décontracté et amical, qui se poursuivait autour d'un repas et de quelques bonnes bouteilles.

Un autre élément moteur, pour moi, a été l'ouverture comparatiste. Je suivais en cela l'exemple de mes maîtres, Detienne et Vernant. Cette ouverture comparatiste a été rendue possible par un ensemble de rencontres et de solides amitiés : je citerai Jean-Jacques Glassner, un assyriologue élève de Bottéro, qui a contribué à renouveler les approches de l'écriture et de la divination en Mésopotamie, mais aussi Jean-Pierre Drège, directeur d'études à l'EPHE, spécialiste de l'histoire du livre et des bibliothèques en Chine, Anne Cheng, historienne de la pensée chinoise, Viviane Alleton, directrice d'études à l'EHESS, et ses travaux sur l'écriture et la langue chinoises, Luce Giard, spécialiste d'histoire des sciences, d'histoire de la spiritualité jésuite, devenue une amie proche, Charles Malamoud sur l'Inde, ainsi que des collègues sanskritistes plus jeunes, Gérard Colas et Cezary Galewicz, Gilles Dorival sur la tradition biblique, Guy Stroumsa, sur la tradition chrétienne et juive, Javier Teixidor sur les milieux syriaques, Alfred-Louis de Prémare, sur l'écriture du Coran, Chloé Ragazzoli, sur les pratiques lettrées en Égypte ancienne. Ce sont les principaux noms qui me reviennent à l'esprit, il y eut bien sûr d'autres collègues, rencontrés dans des colloques, écoutés dans des séminaires, mais aussi dont j'ai lu les travaux.

Les deux colloques Des Alexandries ${ }^{39}$, organisés à la demande conjointe de la Bibliothèque nationale de France et de la Bibliotheca Alexandrina m'ont donné l'occasion de lire beaucoup de littérature secondaire sur les grandes cultures lettrées non européennes, l'Islam, la Chine, le Japon, I'Inde, la tradition juive : je me suis intéressé en particulier à l'histoire des bibliothèques et du livre, à la constitution des corpus canoniques, aux commentaires, à l'histoire de la philologie et de l'édition textuelle. Je me suis d'ailleurs constitué un fonds de livres sur ces sujets, ainsi qu'une petite collection de sources primaires en traductions française ou anglaise, comme le Fihrist de al-Nadim et un certain nombre de classiques chinois.

Ma pratique du comparatisme, suivant en cela l'exemple de Marcel Detienne ${ }^{40}$, suppose à la fois de s'entourer d'un réseau de collègues compétents, maîtrisant les langues et les sources de première main, mais aussi de faire l'effort personnel d'un ensemble de lectures permettant une première appréhension de ces traditions culturelles et de leurs bibliothèques lettrées. En d'autres termes, ce qui $\mathrm{m}^{\prime}$ intéressait dans le comparatisme était la capacité de rapprocher, de confronter, d'interroger ces traditions les unes par rapport aux autres, y compris avec celles dont j'étais plus familier. La comparaison était pour moi non la recherche d'invariants ou d'universaux, ni la simple déclinaison des différences, mais une incitation à comprendre des processus, des dynamiques culturelles, des pratiques lettrées et savantes dans leur matérialité et leur formalité, ainsi que tous les paramètres contextuels, sociaux, politiques, religieux, techniques, qui leur donnent des significations, des fonctions et une portée différentes d'une culture à l'autre.

\footnotetext{
${ }^{37}$ Bruno Latour, La science en action, trad., Paris, La Découverte, 1989.

${ }^{38}$ Bruno Latour et Steve Woolgar, La vie de laboratoire. La production des faits scientifiques, trad., Paris, La Découverte, 1988.

${ }^{39}$ Christian Jacob et Luce Giard (dir.), Des Alexandries, T. I : Du livre au texte, Paris, Bibliothèque Nationale de France, 2001 ; Christian Jacob (dir.), Des Alexandries, T. II : Les métamorphoses du lecteur, Paris, Bibliothèque Nationale de France, 2003.

${ }^{40}$ Marcel Detienne, Comparer l'incomparable, op. cit.
} 
Comme Detienne, j'en suis venu à augmenter la part d'expérimentation dans cette pratique comparative, nourrie par le dialogue ou par les lectures. D’abord cantonnée dans le cadre des pratiques et des traditions lettrées, la comparaison s'est considérablement élargie, par exemple pour le projet des Lieux de savoir, où l'écart maximal était parfois recherché pour mieux aider au processus de déconstruction et de reconstruction des objets étudiés.

\section{Politique des études grecques}

Zilsel - Les études grecques supposent un rapport philologique quasi maniaque aux textes. Comment t'es-tu positionné vis-à-vis de cette exigence disciplinaire?

Christian Jacob - Je ne me définis pas comme un philologue et je n'en revendique pas la technicité, par exemple en termes de travail sur les manuscrits, d'établissement du texte, même si, dans ma jeunesse, j'ai publié une traduction de la Description de la terre habitée de Denys d'Alexandrie ${ }^{41}$. Certes, ma formation en lettres classiques me permet un accès direct aux textes grecs et latins comme à l'ensemble des instruments de travail qui peuvent les éclairer. Et j'ai respect et reconnaissance pour les collègues qui produisent ces éditions et ces instruments comme les commentaires, sans lesquels mon travail d'historien serait impossible.

Je crois que mon approche des sources anciennes repose sur l'interprétation, et plus précisément, sur un souci de problématisation et d'intelligibilité. II m'arrive de lire des textes selon des angles thématiques particuliers, mais le plus souvent, j'aime lire les textes pour comprendre leur projet, leur dynamique interne, leur fonctionnement. Je les considère au fond comme des lieux de savoir, et j'essaie d'observer les acteurs qui y opèrent, leurs outils et leurs manières de faire, les artefacts qu'ils produisent.

Qu'il s'agisse de Pausanias, d'Athénée, de Strabon ou de Denys le Périégète, d'Aulu-Gelle ou de Pline l'Ancien, ce qui m'intéresse, c'est l'atelier. C'est toujours pour moi une image extraordinairement concrète, un éclairage heuristique sur le texte ou le document. C'est aussi ce que j'essaie de faire comprendre à mes étudiants : quand on travaille sur une archive, un texte, une correspondance, un corpus, des images, il faut déconstruire ces artefacts, faire du retro engineering et regarder comment $c^{\prime}$ est fait, comment cela fonctionne. Par exemple, j'ai écrit autrefois une introduction au livre II des Histoires d'Hérodote, consacré à l'Égypte ${ }^{42}$. J'ai essayé de faire vivre ce texte, en faisant porter mon éclairage sur cet auteur grec formé à la rationalité ionienne, qui se promène en pays pharaonique avec la trousse à outils de cette rationalité. II a une règle, un compas, mais aussi des outils pour comparer, pour construire des analogies. II a une carte de la terre, même s'il se montre très critique à son égard. II a aussi son savoir sur les dieux grecs, qu'il va confronter à celui des prêtres égyptiens. Et selon les gens qu'il va rencontrer, les endroits qu'il va visiter, il va sortir certains outils de sa trousse, les appliquer, se demander si cela marche ou non pour penser l'altérité de ce pays et la rendre compréhensible à des lecteurs et auditeurs de langue grecque. Une telle hypothèse de lecture conduit à observer des opérations, des pratiques, bref, un véritable bricolage conceptuel et discursif. J'ai appliqué ce protocole de lecture aux Deipnosophistes d'Athénée, aux Nuits Attiques d'Aulu Gelle ${ }^{43}$, à la Géographie de Strabon comme aux fragments des généalogistes et logographes grecs ${ }^{44}$. Mon approche des textes grecs a inspiré le concept de lieux de savoir, mais s'est aussi évidemment enrichie du travail interdisciplinaire mené dans ce cadre.

\footnotetext{
${ }^{41}$ Denys le Périégète, La description de la terre habitée de Denys d'Alexandrie ou la Leçon de géographie, introduit et traduit par Christian Jacob, Paris, Albin Michel, 1990. Nouvelle édition en cours pour la collection "La Roue à livres ", aux Belles Lettres.

42 Hérodote, L'Égypte, Histoires II, introduction de Christian Jacob, traduction de Philippe-Ernest Legrand, Paris, Les Belles-Lettres, 1997.

43 Christian Jacob, " "La table et le cercle". Sociabilités savantes sous l'Empire romain ", Annales. Histoire, Sciences Sociales, $60^{\mathrm{e}}$ année, $\mathrm{n}^{\circ} 3,2005$, p. 507-530.

${ }^{44}$ Voir Christian Jacob, "L'ordre généalogique. Entre le mythe et l'histoire ", in Marcel Detienne (dir.), Transcrire les mythologies. Tradition, écriture, historicité, Paris, Albin Michel, 1994, p. 169-202 ; Christian Jacob, Géographie et ethnographie en Grèce ancienne, Paris, Armand Colin, $2^{\mathrm{e}}$ édition revue et augmentée, 2017.
} 
Zilsel - Dans un contexte très difficile pour les sciences humaines et sociales, les études grecques souffrent davantage d'une désaffection politique. Comment vois-tu leur futur?

Christian Jacob - En tant qu'enseignant-chercheur on est bien évidemment partagé entre un désir de formation et de transmission et une exigence de réalisme qui fait qu'on hésite à lancer des étudiants dans la voie longue d'un doctorat, qu'il s'agisse d'études anciennes ou d'autres disciplines des sciences humaines, dont les débouchés professionnels sont incertains et aléatoires, et se raréfient de plus en plus. Quel discours tenir à un ou une étudiant.e qui voudrait s'engager aujourd'hui dans une thèse d'histoire ou de littérature grecques ? Les postes de maîtres-se de conférence en université, lorsqu'il y en a, attirent une foule des candidatures. De même au CNRS, où les postes de chargé.e.s de recherche sont créés au compte-gouttes, souvent fléchés, et donnent lieu à une concurrence acharnée et souvent imprévisible (il y a cinquante candidatures pour un poste). II faut mesurer la motivation des étudiants, mais aussi leurs atouts, leurs chances de parvenir au terme d'un doctorat, leur formation technique, mais aussi l'existence d'un « plan B » qui pourrait leur assurer un gagnepain dans l'attente d'hypothétiques bourses post-doctorales ou d'un premier recrutement. Le Certificat d'Aptitude au Professorat du Second Degré (CAPES) ou l'agrégation apportent une certaine sécurité, même si la contrepartie est que la préparation de la thèse doit alors s'accommoder d'une charge d'enseignement dans le secondaire. Les contrats doctoraux financés sont rares, la concurrence ici encore est féroce. Plusieurs de mes doctorants ont obtenu des postes en université, j'en suis ravi. Un autre, un antiquisant, est professeur d'histoire dans le secondaire.

À l'EHESS, faire de l'histoire ancienne se pose aussi dans des termes un peu particuliers. On n'est pas dans une filière universitaire classique, par exemple Paris IV ou Paris I. On est dans un établissement tourné vers la recherche en sciences sociales, riche, il est vrai, d'une belle tradition dans le champ des études anciennes, avec des collègues comme Nicole Loraux, Pierre Vidal-Naquet, François Hartog, Claude Calame, Pierre de la Combe, François Lissarrague, pour ne citer que quelques noms. Ce n'est pas faire injure à mes collègues actuels que de dire que les études anciennes n'ont plus le rayonnement qu'elles ont pu avoir à la grande époque de nos maîtres. Nous avons toujours des étudiants, néanmoins, notamment étrangers. À quoi servent le grec et le latin à l'EHESS ? L'Antiquité classique constitue un " terrain " au même titre que d'autres sociétés et cultures anciennes ou modernes, proches ou lointaines. On peut, en étudiant ce terrain, contribuer à éclairer des questions plus générales et parfois très actuelles, qu'il s'agisse du politique, du religieux, de l'altérité, du genre, de l'image, des techniques, des savoirs et des sciences. De ce point de vue, si nous pouvons encadrer des étudiants qui veulent se spécialiser sur ce terrain, notre rôle est aussi d'y introduire des étudiants travaillant sur d'autres aires et d'autres époques, désireux d'expérimenter le décentrement comparatiste. À titre personnel, je ne me définis pas, ou plus, comme "spécialiste de l'Antiquité ", et mon enseignement, s'il s'appuie souvent sur ce terrain, le dépasse largement. Ce sont les questions, les problématiques, la transdisciplinarité liée à I'histoire et à l'anthropologie des savoirs qui structurent mes séminaires, et en élargissent évidemment le public...

Zilsel - Pourtant les études antiques apportent leur lot de nouveautés. Et l'on ne cesse de (re)découvrir des textes, des versions inédites ou méconnues. Comment s'opère ce travail de découverte sur l'Antiquité ?

Christian Jacob - Qu'est-ce qui fait la valeur, le caractère novateur d'un travail en sciences humaines et sociales ? Dans les sciences dures, on peut parler de la résolution d'un problème, de la découverte d'une molécule, d'un vaccin, d'une nouvelle étoile, d'un nouveau modèle de compréhension de l'univers physique, d'une potentielle application industrielle, etc. Dans nos disciplines, l'innovation revêt une forme différente. On peut penser à la découverte et à l'édition d'une source inconnue, d'un texte nouveau, par exemple le traité de Galien Ne pas se chagriner, qui fut découvert fortuitement par Antoine Pietrobelli dans une bibliothèque de monastère grec ${ }^{45}$. II s'agit d'une découverte spectaculaire qui ne doit pas dissimuler toutes

\footnotetext{
${ }^{45}$ Galien, CEuvres, T. IV : Ne pas se chagriner, texte établi et traduit par Véronique Boudon-Millot et Jacques Jouanna, contribution d'Antoine Pietrobelli, Paris, Les Belles Lettres, 2010.
} 
celles qui ne retiennent que l'attention du cercle des spécialistes : inscriptions, fragments de papyrus qui parfois révèlent des textes inédits. À cette même catégorie appartiennent des éditions savantes, critiques et commentées, qui construisent la lisibilité et l'intelligibilité d'un texte, ou en offrent des traductions nouvelles. Je pense en particulier à la traduction du corpus platonicien dans la collection Garnier Flammarion, sous l'égide de Luc Brisson et d'un cercle de spécialistes.

Une seconde forme d'innovation réside dans des livres-somme qui renouvellent l'étude d'un domaine ou parfois le fondent. On peut penser à certains grands livres de Michel Foucault, L'histoire de la folie, Surveiller et punir, L'histoire de la sexualité, Les mots et les choses ${ }^{46}$, etc. Même si on peut discuter certaines des interprétations, ces livres sont devenus des classiques, pris dans de multiples circulations, qui construisent un champ et apportent des outils critiques, des modèles théoriques pour l'exploiter. De mon point de vue, les grands livres, ou des articles " séminaux " dans nos disciplines conjoignent ces deux dimensions, empirique et théorique, renouvelant la compréhension, la perception d'un champ ou d'un objet. Pour revenir à mes maîtres, je dois dire que tous leurs livres ont été marqués, pour moi, du sceau d'une innovation absolue par rapport à l'état de la question, aux interprétations dominantes ou à l'absence de toute problématisation sur ces objets jusqu'alors. Les chapitres de Mythe et pensée chez les Grecs, des Jardins d'Adonis, de Ruses de l'intelligence restent pour moi des ovnis dans le champ des études anciennes, même si Detienne et Vernant ont fait école et que plus personne ne se risquerait aujourd'hui à disqualifier leurs travaux.

On pourrait sans doute dire la même chose d'un certain nombre de livres de Pierre Bourdieu, de Bruno Latour, Michel de Certeau, et autres, pour m'en tenir à des "classiques". Si je voulais préciser ma pensée, je dirais que ces livres offrent encore aujourd'hui des voies de questionnement, des outils méthodologiques, des concepts opératoires, que l'on peut adapter, appliquer à de nouveaux objets. Que l'on pense aux résonances de l'œuvre de Foucault dans de multiples sous-branches des sciences sociales aujourd'hui. Ou de l'impact du chapitre de Michel de Certeau sur la lecture comme braconnage sur l'histoire des pratiques culturelles et du livre, avec les travaux de Roger Chartier et tant d'autres.

La nouveauté, toutefois, peut aussi prendre des formes plus modestes, mais non moins réelles: le choix d'un nouvel objet, d'un nouveau terrain; la construction d'une question ; l'application d'une nouvelle méthodologie à une question ou à un objet déjà bien connus. Il y a aussi les effets propres à une analyse, une interprétation, une description, un récit dont l'écriture, le choix des mots, l'acuité de la formulation seraient porteurs d'une nouvelle intelligibilité de l'objet traité.

En d'autres termes, ce qui fait la valeur et le caractère innovant d'un travail en sciences humaines et sociales aujourd'hui, c'est la conjonction d'un objet, d'une technicité mise en œuvre dans son traitement, et aussi d'idées nouvelles, provocantes, suggestives, ouvrant des pistes, reconfigurant le champ d'une question.

Notre rôle d'enseignants et d'encadrants est à la fois d'enseigner cette technicité et d'encourager la génération d'idées, l'imagination interprétative, théorique et conceptuelle. Cette dernière peut faire l'objet d'un entraînement, et c'est le propre des travaux les plus riches en idées d'en générer de nouvelles dans l'esprit de leurs lecteurs.

\section{Retrouver les Lieux de savoir}

Zilsel - Au début des années 2000, tu t'engages dans le projet monumental des Lieux de savoir. Quelle en a été la genèse?

Christian Jacob - Le projet a pris forme à la suite des deux volumes Des Alexandries, Du livre au texte et les Métamorphoses du lecteur, où j'avais expérimenté, avec l'aide de Luce Giard

\footnotetext{
${ }^{46}$ Michel Foucault, Les Mots et les Choses. Archéologie des sciences humaines, Paris, Gallimard, 1967 ; id., L'histoire de la folie à l'âge classique, Paris, Gallimard, 1972 ; id., Surveiller et punir. Naissance de la prison, Paris, Gallimard, 1975 ; id., Histoire de la sexualité, T. I : La volonté de savoir, Paris, Gallimard, 1976 ; id., Histoire de la sexualité, T. II : L'usage des plaisirs, Paris, Gallimard, 1984 ; id., Histoire de la sexualité, T. III : Le souci de soi, Paris, Gallimard, 1984 ; id., Histoire de la sexualité, T. IV : Les aveux de la chair, Paris, Gallimard, 2018.
} 
pour le premier ouvrage, la construction de livres collectifs reposant sur le comparatisme, avec des textes d'articulation dégageant les enjeux de chaque partie.

J'avais alors un soutien institutionnel à travers un Groupement de recherche international (GDRI) du CNRS et une Action concertée incitative ( $\mathrm{ACl}$ ) du Ministère de la Recherche, dont les financements permirent l'organisation de colloques et de journées d'étude pendant plusieurs années. Le GDRI s'intitulait " Mondes lettrés », et le projet dans une première étape fut celui d'une histoire comparée des pratiques lettrées. L'élargissement du champ aux pratiques scientifiques comme aux pratiques des sociétés sans écriture a fait débat. Je mesurais bien les multiples différences entre les sciences de la modernité occidentale, reposant sur l'expérimentation, la mathématisation, les laboratoires instrumentés, et les pratiques d'érudition et les modes de sociabilité des mondes savants antérieurs, où chez un Newton, un Leibniz et tant d'autres, on observait un continuum dans l'exercice de l'activité savante plus qu'une rupture nette.

À mes yeux, toutefois, le dépassement de ce clivage présentait l'avantage d'une remise à plat radicale, d'une reconfiguration de l'ensemble du champ des pratiques savantes, en partant du plus concret, les lieux, les mobiliers, la main et les gestes, les instruments et les supports, les opérations techniques, graphiques, discursives, mentales, sociales, la matérialité des artefacts produits, la constitution des collectifs savants, leur institutionnalisation, la ritualisation de leurs activités, et enfin, bien sûr, les procédures de validation des énoncés et des objets ainsi élaborés, investis de valeurs de vérité, de performativité, d'orthodoxie et d'orthopraxie.

Nous avons beaucoup discuté en interne de cet élargissement, de ses risques et de ses bénéfices, notamment lors de séminaires mémorables à la Fondation des Treilles, qui nous a accueillis, si je me souviens bien, trois ans de suite. Il faut s'imaginer des ateliers de travail passionnants et passionnés, qui réunissaient doctorant.e.s et chercheuses et chercheurs de rang international dans une même convivialité... Parmi les éléments qui m’ont décidé, il y a eu un entretien avec Christiane Sinding, médecin et historienne de la médecine, une collègue bienveillante, d'une grande culture en sciences sociales, qui a jugé le projet intéressant, par cette redistribution des frontières entre sciences, humanités, techniques, arts et spiritualités.

Il y a donc eu un véritable laboratoire d'idées, comme un centre de recherche international, mais non institutionnalisé, basé sur des affinités électives, qui a fonctionné pendant plusieurs années, en organisant parfois dix colloques ou workshops par an. Je menais parallèlement un ensemble de lectures dans un ensemble de domaines, histoire et sociologie des sciences, anthropologie, histoire des techniques, philosophie, etc.

Ce projet intellectuel est devenu un projet éditorial suite à une conversation avec Hélène Monsacré, responsable des sciences humaines chez Albin Michel. Nous nous connaissons depuis nos années d'études : elle suivait les cours de Vernant, Detienne, Vidal-Naquet, elle a écrit une thèse originale sur l'épopée homérique, qui a donné lieu au livre Les larmes $d^{\prime} A_{c h i l l e}{ }^{47}$. Donc, je lui parle lors d'une conversation informelle de ce projet d'une histoire comparée et interdisciplinaire des pratiques savantes, sans doute en plusieurs volumes, une entreprise lourde, en lui demandant son avis sur la faisabilité éditoriale. Pas une seconde, je n'ai pensé qu'Albin Michel pouvait être intéressé, même si c'était la maison où j'avais publié mes premiers livres. À ma grande surprise, Hélène Monsacré accroche sur l'idée. "Pourquoi pas ", me dit-elle.

Nous sommes fin 2001 ou début 2002, soit cinq ans avant la parution du volume I des Lieux de savoir. C'est un long processus qui commence. D'une part, l'étude de faisabilité, l'évaluation des coûts, le montage du projet. L'idée est soumise au P.-D.G., Francis Esménard, qui ne la rejette pas d'emblée, mais donne son accord pour qu'on avance dans la réflexion. On travaille à trois, avec Hélène Monsacré, Jacqueline Favero, responsable du développement et des droits étrangers, et moi. Il y a un réel enthousiasme pour ce projet, considéré comme une opération de prestige. Parallèlement à ces discussions, je travaille avec mon réseau sur la construction intellectuelle du projet. L'idée d'un ouvrage en quatre volumes prend corps. Gilles Dorival, spécialiste de la Bible d'Alexandrie (le Pentateuque), donne au projet le nom de code de "Tétrateuque ». Dès le départ, la structure d'ensemble est posée: l'ouvrage ne sera ni

\footnotetext{
${ }^{47}$ Hélène Monsacré, Les larmes d'Achille. Héros, femme et souffrance chez Homère, Paris, Le Félin, 2010.
} 
chronologique, ni disciplinaire, ni organisé par aires culturelles, mais proposera des coupes transversales dans l'histoire des savoirs selon quatre axes thématiques: les lieux et les communautés, les pratiques, les modes de validation, de communication et de transmission, et enfin, dans un quatrième volume, une réintroduction de l'historicité pour penser des processus à l'échelle d'aires culturelles ou de périodes: savoirs de la réflexivité, de I'hybridation, de l'universalité.

Le titre d'ensemble Lieux de savoir est proposé par Hélène Monsacré, si je me souviens bien, en référence aux Lieux de mémoire de Pierre Nora, et je l'accepte aussitôt: "Oui, c'est exactement cela ». Francis Esménard donne le feu vert. Hélène Monsacré me dit : "Un volume sur les savoirs de 300 pages, Albin Michel aurait dit "non", mais quatre volumes de près de 1000 pages, c'est "oui". "

Commence alors la construction du sommaire du premier volume. J'en ai quarante versions successives dans mes archives, de 2004 à 2006. Il faut construire une architecture intellectuelle idéale, avec une progression thématique, mais aussi une pondération entre les périodes, les cultures, les aires géographiques, les disciplines et les traditions. Je m'appuie sur un réseau de spécialistes qui, pour chaque domaine, $m^{\prime}$ indiquent des sujets possibles, des thèmes émergents, et surtout des auteurs potentiels. Vient alors la phase de contact avec ces auteurs, avec une lettre personnalisée à chacune ou à chacun, expliquant le projet mais aussi définissant précisément les contours du chapitre que je leur demandais. Certains auteurs déclinent l'invitation ou proposent un autre sujet, d'autres ne répondent pas. Cela fait bouger le sommaire à chaque fois, il faut procéder à des déplacements et à des substitutions, à l'échelle d'une section ou parfois d'une partie entière.

Ce fut un travail de fou, tant sur le plan de la coordination du collectif d'auteurs (je n'avais pas de secrétariat, j'ai écrit des centaines de mails) que sur celui du travail sur les textes (j'ai tout relu, et souvent partiellement réécrit les textes ou les traductions, qu'il fallait ensuite soumettre aux auteurs pour approbation) et sur la construction de l'armature intellectuelle (l'introduction, les textes d'articulation, etc.). II y eut aussi les relectures et corrections sur épreuves, où Hélène Monsacré et moi avons à nouveau tout relu, après les auteurs.

Zilsel - Les Lieux de savoir devaient constituer une tétralogie. Finalement, seuls deux volumes sont parus. Est-ce une des difficultés de la recherche aujourd'hui que de devoir passer systématiquement sous les fourches caudines d'éditeur aux intérêts divergents de celles et ceux des chercheuses et chercheurs? Comment s'est soldé le différend avec Albin Michel ?

Christian Jacob - Je crois qu'Albin Michel aurait souhaité, dans l'idéal, que les quatre volumes paraissent simultanément ou très rapprochés, pour des raisons de commercialisation et de promotion. Je dois mettre au crédit d'Albin Michel de s'être engagé dans cette aventure hors norme, en me laissant une liberté intellectuelle totale dans la construction des volumes, le choix des sujets et des auteurs, et d'avoir investi des moyens importants : tous les auteurs étaient payés, et assez correctement. II y a eu aussi les frais de traduction, et aussi les droits pour l'iconographie. Et enfin le choix a été fait d'avoir des volumes cartonnés et reliés, avec jaquette en quadrichromie, le premier volume faisait 1280 pages! Nous avons eu quelques subventions (CNL, Fondation des Treilles, CNRS), mais l'investissement était considérable.

Le volume II, Les Mains de l'intellect a été lancé, en suivant le même processus que pour le volume I. Je garde trace de 43 versions du sommaire, les premières remontant en 2004 . La réception critique du premier volume, publié en 2007, fut très positive. Le travail de commande des textes et de construction de ce volume II n'a commencé qu'en 2007, le volume, plus complexe, plus novateur, aussi, est paru en janvier 2011 : sur le conseil des commerciaux, nous avons laissé passer le créneau de mise en vente des fêtes de fin d'année 2010. En vue de baisser les coûts de papier et de reliure, on a modifié la mise en page, moins aérée et élégante que pour le volume I. Le P.-D.G. à un moment a même songé à renoncer à faire imprimer l'ouvrage alors que nous en étions au bon-à-tirer. On m'a demandé de renoncer presque totalement au petit pourcentage de droits d'auteur que je devais avoir sur le volume, ce que j'ai accepté. Le volume a finalement été publié. La réception critique, ici encore, a été bonne (je me souviens d'une invitation dans un talk-show décoiffant sur France Inter, à une heure de grande écoute...). Les ventes des deux premiers volumes, toutefois, si elles ont été 
très correctes pour un ouvrage de sciences humaines et sociales, ont été jugées décevantes par rapport aux investissements consentis par Albin Michel et à leurs attentes commerciales. La suite dès lors était compromise. Après un an de tergiversations, la décision a été prise de ne pas lancer les commandes de textes pour le volume III. On en est resté là. On me donnait le droit de poursuivre le projet chez un autre éditeur, mais Albin Michel se réservait la " marque " Lieux de savoir, ce que j'ai trouvé abusif. Il s'en est suivi un contentieux légal, à coups de courriers d'avocats (j'avais demandé au cabinet Pierrat de me représenter). Cela m'a coûté assez cher, les échanges avec le conseil d'Albin Michel ont été de plus en plus violents (pour moi). Je ne suis pas allé jusqu'au procès.

Le sort des Lieux de savoir est resté en stand-by, jusqu'à ce que le départ de Francis Esménard et l'arrivée d'un nouveau P.-D.G., Gilles Hoeri, débloquent la situation : au printemps 2018, Hélène Monsacré et la Secrétaire générale d'Albin Michel, Agnès Fruman, avec l'accord du P.D.G., m'ont cédé gracieusement les droits numériques des textes des Lieux de savoir pour une publication en open access sur la plateforme Savoirs. Et je leur en suis vivement reconnaissant. C'était impensable il y a quelques années...

Je peux aujourd'hui reconsidérer cet immense chantier avec sérénité et en tirer quelques leçons. D'abord, je suis fier du travail effectué, du réseau que j'ai coordonné, et de ces deux volumes qui, je crois, continuent à circuler et peut-être à inspirer. Et je suis reconnaissant à Albin Michel d'avoir soutenu ce projet et mené à bien la publication de deux volumes, qui, j'en suis conscient, ont représenté un fort investissement financier. Je reconnais que je n'ai pas pu mener la publication des quatre volumes dans un délai plus serré : un ou deux ans d'intervalle. La charge de travail et de coordination était trop lourde, je n'avais pas les soutiens en termes de secrétariat. La complexité du tissage comparatiste et interdisciplinaire, de l'identification des bons sujets et des bons auteurs, et les inévitables retards dans la réception des chapitres commandés ont aussi joué leur part dans ce délai. Pour le volume II, outre l'introduction générale, j'ai écrit quatre textes d'articulation, pendant l'été 2010 , sous une forte pression, et dans un état de grand épuisement intellectuel.

Je pense rétrospectivement qu'il y avait deux logiques difficilement conciliables: celle de l'éditeur, avec ses objectifs commerciaux, et aussi sa stratégie de prestige, avec un projet ambitieux, positionné dans la catégorie des "livres de référence ", voire des " beaux livres ", un projet qui faisait pendant aux Lieux de mémoire de Gallimard. Et ma logique de chercheur et d'intellectuel, qui demandait le temps de la réflexion, de la construction, un gros travail préparatoire de cartographie des objets, des problèmes, des sujets et des auteurs possibles. Le titre du deuxième volume, Les mains de l'intellect était porteur d'une proposition théorique ambitieuse, et s'approchait des sciences cognitives sans toutefois sortir du champ de l'histoire et de l'anthropologie des savoirs. Je continue à creuser cette proposition dans mon travail personnel actuel.

Lorsque le projet a été lancé, en 2002, nous étions sur le modèle d'une publication imprimée, avec des contrats où chaque auteur cédait tous les droits de son texte, urbi et orbi, moyennant une indemnité forfaitaire. On avait pensé à une déclinaison numérique, mais Albin Michel ne s'est jamais engagé sur cette voie.

Le format imprimé, d'autre part, figeait et fossilisait un projet évolutif et fluide dans un sommaire "définitif", dans un réseau de connexions stabilisé et d'une certaine façon désactivé. Sans doute que l'état des technologies numériques, comme les modèles de l'édition traditionnelle, ne permettait pas d'envisager d'autres scénarios à l'époque. Aujourd'hui, la problématique du libre accès et les nouveaux écosystèmes numériques de l'édition scientifique offrent d'autres modèles : ceux-ci auraient peut-être permis de rendre le projet des Lieux de savoir économiquement viable, mais surtout de le repenser sur des bases entièrement nouvelles. C'est au fond ce sur quoi je travaille depuis l'an dernier, dans le cadre du projet Savoirs.

\section{Savoirs, une plateforme pour distribuer la connaissance}

Zilsel - La plateforme Savoirs prend en quelque sorte le relais de la version papier. Comment envisages-tu à la fois l'inscription dans une généalogie qui était celle des Lieux de savoir et le déploiement de nouvelles opportunités permises par le numérique? 
Christian Jacob - Après le retrait d'Albin Michel, j'ai pour un temps voulu poursuivre le plan initial, réaliser ce volume III, intitulé La pragmatique des savoirs, puis le volume IV, Réflexivité, hybridation, universalité. II y a eu un certain nombre de sommaires préparatoires pour le volume III, des réunions et des séminaires de réflexion, notamment avec un petit cercle de collègues du Centre Alexandre Koyré, auquel s'étaient joints des doctorants.

La véritable difficulté résidait dans le choix du modèle éditorial, et bien sûr de l'éditeur. L'option numérique s'est rapidement imposée, des contacts ont été pris notamment avec les Éditions de l'EHESS, les Presses de la Sorbonne et OpenEdition. Mais on restait sur le modèle d'un livre "classique ", au sommaire stabilisé, et disponible sous forme d'ebook, en format .epub, .pdf, ou .html. Des tentatives amiables ont été faites, notamment avec le service juridique de l'EHESS, pour obtenir le droit d'utiliser le titre Lieux de savoir: nous n'avons jamais eu de réponse d'Albin Michel, puis un jour, on nous a demandé notre modèle économique, ce qui était une façon de différer la réponse sur le fond.

Je n'ai jamais tout à fait renoncé à l'idée de relancer le projet, même s'il fallait réinventer la forme, les moyens, l'équipe de ces Lieux de savoir 2.0. Le soutien et l'incitation d'un petit cercle amical, parmi lesquels Jean-François Bert, ont été les éléments déclencheurs en mai-juin 2017.

Savoirs n'est pas à proprement parler la suite des Lieux de savoir. C'est un autre concept, un autre projet, intellectuel, éditorial et technologique. II s'agit d'une bibliothèque nativement numérique, mais qui ne se réduit pas à une collection de textes indépendants et consultables comme sur les revues en lignes habituelles. Cette bibliothèque doit permettre des parcours exploratoires et heuristiques parmi les textes, sur la base de requêtes croisées et paramétrables par l'utilisateur. Ces requêtes s'appuient sur une indexation fine des articles sur différents critères : dates, lieux géographiques et concepts, et permettent donc de dérouler des fils analogiques pour explorer une question, un problème, un champ de concepts en sortant du cadre de sa spécialité disciplinaire. C'est donc une bibliothèque qui met en œuvre le comparatisme expérimental naguère théorisé par Marcel Detienne, en partant du principe que la confrontation d'études de cas traitant des mêmes questions, mais dans des périodes et des aires géographiques différentes, peut élargir l'horizon d'une recherche, multiplier les éclairages, enrichir la problématisation.

Il y a plusieurs dimensions à ce projet. Une dimension intellectuelle, bien sûr, par son hypothèse comparatiste et interdisciplinaire, qui présuppose que les chercheurs en sciences humaines et sociales ne peuvent que tirer bénéfice du décentrement et de la mobilité pour introduire de la complexité, de la multidimensionnalité dans leurs objets de recherche. Une dimension éditoriale, aussi. Dans cette phase de construction, la bibliothèque Savoirs accueille trois ensembles de textes en open access : tous les textes des deux volumes imprimés des Lieux de savoir, suite à l'accord avec Albin Michel; une sélection de textes provenant de différentes revues d'OpenEdition (avec accord des auteurs et des comités éditoriaux) ; les communications inédites présentées au colloque Spectres de l'érudition organisé à Lausanne en 2017. Il en résulte un corpus assez ouvert et diversifié, qui devrait dépasser les deux cents textes au début 2020. Une dimension technologique, enfin. Car le projet implique la conception et le développement d'une application originale, avec un back-office et une interface utilisateur accessible sur le Web. Cette phase est actuellement bien engagée, et une équipe de développeurs et ingénieurs travaille sur ce chantier.

De cette expérience, je voudrais tirer quelques observations. II est possible de monter un projet innovant et ambitieux sans passer par les appels d'offres ANR et ERC, qui peuvent offrir, il est vrai, des financements considérables, mais avec des structures administratives lourdes, et surtout un taux de succès relativement bas. Cela ne nous a pas empêchés de répondre à des appels à financements plus modestes, mais dans des structures et selon des procédures «à taille humaine " (LabEx Hastec et Fondation de la Maison des Sciences de l'Homme). Ensuite, un projet de ce type ne peut réussir qu'au prix d'une convergence de compétences, de métiers et d'expériences. L'un des points très positifs, de mon point de vue, réside dans les synergies qui se sont nouées entre des ingénieurs informaticiens, un développeur free-lance, des professionnels de l'édition numérique, des conservateurs de bibliothèques, des enseignant-e-schercheuses.rs, des étudiant.e.s masterant.e.s, doctorant.e.s et des post-docs, sans oublier 
tous les agent.e.s administratifs, les gestionnaires et les représentant.e.s des différentes tutelles impliquées. Certains ont consacré du temps et du travail à Savoirs dans le cadre de leur activité professionnelle, d'autres ont été rémunérés pour leur participation (sous forme de contrats à durée déterminée, d'indemnité de stage ou de paiement sur facture), d'autres se sont investis bénévolement, par conviction personnelle. Chacune et chacun a joué un rôle dans ce projet collectif, complexe, dont chaque étape a fait l'objet de concertations, de pondération, de feuilles de route élaborées collectivement. Ces synergies ont impliqué un vrai travail collaboratif, où nous avons pu communiquer au-delà de la technicité des objets, par exemple entre informaticiens et chercheurs en sciences humaines et sociales. L'élaboration de cette plateforme numérique innovante serait du reste un objet d'observation passionnant pour une ethnographie des savoirs et des techniques : comment définit-on les spécifications fonctionnelles d'une application? Comment s'opèrent les arbitrages technologiques? Comment procède-t-on aux premières simulations d'écrans (mockup) ? Pourquoi choisit-on de coder dans tel ou tel langage informatique ? Comment se négocient les étapes, les inflexions du développement, lors des réunions de "sprint", au cœur de la "méthode agile " ou "scrum " ? II y aurait aussi beaucoup à dire sur le rôle stratégique des gestionnaires et personnels administratifs qui peuvent être des facilitateurs à toutes les étapes du processus, mais aussi des points de blocage, parfois en raison de la lourdeur des procédures ou simplement la force d'inertie des services et des bureaux. Faute d'un appui en termes de secrétariat, j'assure tout ce travail de coordination : nous avons eu en 2019 quatre guichets de financement relevant de quatre administrations différentes... Le temps passé, le nombre d'emails répétés pour des démarches relativement simples prennent une part non négligeable du quotidien d'un chercheur coordonnant ce type de projet... Ces difficultés réelles, souvent déprimantes, ne doivent pas dissimuler le rôle de toutes celles et tous ceux qui, avec intelligence, générosité et efficacité, font avancer le projet, débloquent les situations, mettent des personnes en relation. Que ce soit à I'EHESS, à l'École Nationale des Sciences de I'Information et des Bibliothèques (ENSSIB), à l'École Polytechnique Fédérale de Lausanne ou à la Bibliothèque Nationale et Universitaire de Strasbourg, j'ai trouvé des interlocuteurs, des soutiens qui ont permis au projet Savoirs de prendre forme et corps...

Zilsel - Est-ce que la plateforme renouvelle vraiment le format du discours savant ? Est-ce qu'il y a davantage d'horizontalité que dans un ouvrage papier?

Christian Jacob - Au moment où je réponds à cette question (novembre 2019), la plateforme Savoirs est en cours de développement, avec différents chantiers qui avancent parallèlement et vont converger dans les prochaines semaines. Je ne peux donc pas encore répondre à cette question, sinon assurer que le concept du projet est d'offrir une expérience de lecture et de réflexion radicalement différentes de ce que l'on peut éprouver face à un ouvrage papier, ou même face à un e-book.

Savoirs est une bibliothèque modulaire, qui invite à des parcours toujours renouvelés en fonction des requêtes des lecteurs. C'est une bibliothèque qui invite à lire ce qu'on n'avait pas prévu de lire, mais dont la lecture s'avérera pertinente dans un projet de recherche, un questionnement, une recherche de parallèles, une hypothèse comparatiste. C'est donc un instrument heuristique, un peu comme un instrument expérimental dans le laboratoire d'un scientifique. Chaque requête, chaque texte sélectionné entraîneront des réactions, des rétroactions, des reconfigurations de la bibliothèque, pour un lecteur particulier, à un moment précis de son cheminement. En d'autres termes, ce lecteur sait quel sera son point d'entrée dans la bibliothèque, mais il ne sait pas quelles seront les étapes suivantes ni quel sera son point de sortie.

Et lors d'une nouvelle visite, le parcours de ce même lecteur sera sans doute totalement différent.

Cette expérience ne repose pas sur le hasard, mais sur des algorithmes qui pondèrent différents critères de recherche et ouvrent des connexions non pas avec un seul texte, mais avec un ensemble de textes susceptibles d'intéresser le lecteur, selon qu'il privilégie les critères géographiques, chronologiques ou conceptuels, ou qu'il souhaite les croiser.

On sait que la bibliothèque imaginée et réalisée par Aby Warburg à Londres partait du postulat que la juxtaposition réfléchie de livres très différents sur une même étagère pouvait avoir un 
rôle heuristique, établir des connexions insoupçonnées, dérouler les fils d'une nouvelle forme d'histoire des représentations, des savoirs, des croyances, des images, des savoirs et des imaginaires. Le " bon voisinage " des bons textes, sur une étagère, avait un rôle génératif dans un projet de pensée et d'écriture.

Toutes proportions gardées, et avec les possibilités offertes aujourd'hui par les technologies numériques, par les algorithmes de recherche et les modes de visualisation des résultats, on peut s'inspirer de ce pouvoir des rayonnages "intelligents " de la bibliothèque de Warburg, pour offrir aux lecteurs un outil de découverte, de dépaysement, d'expérimentation intellectuels.

Un ou une spécialiste d'égyptologie, des études grecques, de science studies, d'anthropologie africaine, ne trouvera pas dans Savoirs des articles relevant de sa discipline qu'il ou elle ne pourrait se procurer ailleurs (quoique...). En revanche, à partir de cet ancrage disciplinaire, de ce point de départ, il ou elle pourrait être conduit.e vers des textes relevant d'autres domaines, même très éloignés, mais apportant néanmoins un éclairage, un parallèle, un élément de comparaison, un outil méthodologique, un développement historiographique ou théorique pertinent sur un sujet relevant de sa spécialité.

Pour que cela fonctionne, il faut qu'un ensemble de conditions soient réunies : une masse critique de textes, suffisamment différents, mais connectables en fonction de leurs questionnements et de leurs objets; une indexation fine des textes qui, dans cette première phase, est opérée manuellement, selon les protocoles de la TEI, à partir de référentiels géographiques et chronologiques, mais aussi d'un thésaurus conceptuel cartographiant, sous forme d'arborescences raisonnées, le champ des savoirs, des sciences et des techniques. Une étape à venir s'appuiera sur l'intelligence artificielle et sur des algorithmes avancés de text mining (notamment par le biais d'un lien avec l'application Gargantext développée par I'Institut des systèmes complexes à Paris).

Il s'agit au fond d'externaliser et d'objectiver un ensemble d'opérations au cœur de l'activité intellectuelle, liée à la digression, à la corrélation, à l'imagination théorique, à la conceptualisation. Non pas, dans une illusion techno-scientiste pour les émuler, mais pour les équiper, pour les instrumenter, en introduisant une dimension réflexive et expérimentale qui accompagnera ces opérations, en fera des exercices de pensée et de conceptualisation basés sur des associations d'idées conduisant de texte en texte, à travers les périodes et les aires géographiques, en suivant les « lignes d'erre » des concepts. 